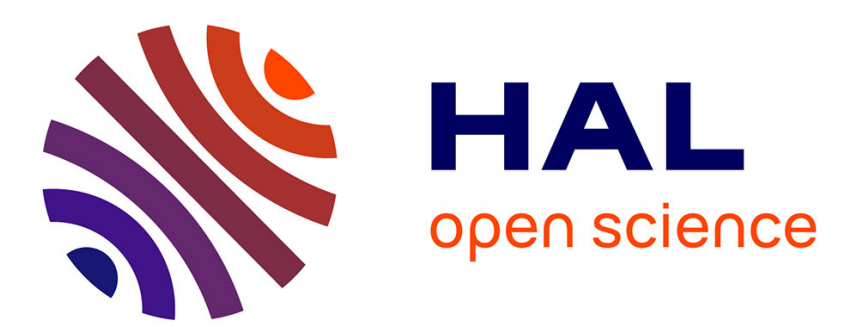

\title{
A non-parametric probabilistic model for ground-borne vibrations in buildings
}

\author{
Maarten Arnst, Didier Clouteau, Hamid Chebli, Ramzi Othman, Geert \\ Degrande
}

\section{- To cite this version:}

Maarten Arnst, Didier Clouteau, Hamid Chebli, Ramzi Othman, Geert Degrande. A non-parametric probabilistic model for ground-borne vibrations in buildings. Probabilistic Engineering Mechanics, 2006, 21 (1), pp.18-34. 10.1016/j.probengmech.2005.06.004 . hal-01004917

\section{HAL Id: hal-01004917 https://hal.science/hal-01004917}

Submitted on 3 Jun 2017

HAL is a multi-disciplinary open access archive for the deposit and dissemination of scientific research documents, whether they are published or not. The documents may come from teaching and research institutions in France or abroad, or from public or private research centers.
L'archive ouverte pluridisciplinaire HAL, est destinée au dépôt et à la diffusion de documents scientifiques de niveau recherche, publiés ou non, émanant des établissements d'enseignement et de recherche français ou étrangers, des laboratoires publics ou privés.

\section{(c)(1)}

Distributed under a Creative Commons Attribution| 4.0 International License 


\title{
A non-parametric probabilistic model for ground-borne vibrations in buildings
}

\author{
M. Arnst ${ }^{\mathrm{a}, \mathrm{b}}$, D. Clouteau ${ }^{\mathrm{a}}$, H. Cheblia ${ }^{\mathrm{a}}$, R. Othman ${ }^{\mathrm{a}}$, G. Degrande ${ }^{\mathrm{b}}$ \\ ${ }^{a}$ MSSMat, UMR8579, Ecole Centrale Paris, Grande Voie des Vignes, F-92295 Châtenay-Malabry Cedex, France \\ ${ }^{\mathrm{b}}$ Department of Civil Engineering, K.U. Leuven, Kasteelpark Arenberg 40, B-3001 Leuven, Belgium
}

\begin{abstract}
This paper studies the prediction accuracy of models for ground-borne vibrations in buildings based on a three-dimensional coupled FE$\mathrm{BE}$ formulation in the frequency range relevant for traffic induced vibrations. In structural dynamics, the prediction accuracy at relatively high frequencies is known to be problematic since the sensitivity of the predicted response to modelling errors and parameter uncertainties increases with the frequency. To estimate the prediction accuracy, this paper incorporates the parameter uncertainties and the modelling errors into the analysis using the non-parametric probabilistic approach, introduced by Soize. The methodology is applied to a case history. The results of a prediction model for the transmission of vibrations from a shallow cut-and-cover tunnel to a six storey reinforced concrete frame structure in Paris are considered and compared with in situ measurements. The results demonstrate that a single dispersion parameter allows fitting the data. The sensitivity of the response to uncertainties is shown to increase as vibrations propagate inside the building.
\end{abstract}

Keywords: Soil-structure interaction; Non-parametric probabilistic model; Prediction accuracy; Tunnel; Building; Coupled FE-BE formulation

\section{Introduction}

Ground-borne traffic induced vibrations in buildings are a major environmental concern in urban areas. Advanced numerical models have been developed to give a valuable insight and quantification of the transmission of vibrations to buildings. Models have been proposed to compute the wave field radiated into the soil due to road [1], railway [2] and underground railway [3] traffic. Dynamic soil-structure interaction models are subsequently used to compute the response of the buildings. These models can be based on a three-dimensional coupled FE-BE formulation and on the Craig-Bampton substructuring method [4], as well established in the field of earthquake engineering [5,6]. Whereas computations for earthquake engineering problems are performed in the frequency range between 0 and $10 \mathrm{~Hz}$, the quantification of traffic induced re-radiated noise in buildings requires computations of the structural vibrations up to $200 \mathrm{~Hz}$. The prediction accuracy at such high frequencies may be problematic since the sensitivity of the predicted response to parameter uncertainties and modelling errors increases with the frequency. As buildings are large and complex structures, parameter uncertainties are always present. In most cases, only simplified models restricted to the main structural parts can be envisaged which introduces significant modelling errors. The aim of this paper is to account for such uncertainties and errors in the estimation of the prediction accuracy of dynamic soilstructure interaction models based on a coupled FE-BE formulation and on the Craig-Bampton substructuring method.

At low frequencies, numerical models allow efficient predictions of ground-borne vibrations in buildings. Their efficiency relies on the fact that the low frequency response of a building is governed by only a few global eigenmodes. The sensitivity of the model response to small perturbations of the mass or stiffness of the building is usually low enough to restrict the uncertainty on the low frequency response to small values. When updated eventually by appropriate 
methods [7], the model predictions usually compare very well with experimental data.

In the medium frequency range, the vibration of buildings is characterized by the superposition of some global eigenmodes and clusters of local eigenmodes [8]. When the latter contribute to the response, parameter uncertainties and modelling errors play a fundamental role. Small perturbations of the mass or stiffness of the structural elements strongly modify the individual local eigenvectors, but leave the space spanned by these eigenvectors basically unchanged [9-11]. Such perturbations may induce strong local modifications of the response.

A number of studies have been reported on the efficient analysis of vibrations in the medium frequency range. A first class of approaches extends the usual numerical methods for low frequency predictions such as the finite element method to the medium frequency range. Adhikari and Manohar [12] have adopted a frequency adaptive basis; Dey et al. [13] have used higher order finite elements and Ben Dhia [14] has used multiscale finite elements.

A second class of approaches are hybrid methods that combine the methods for the high frequency predictions (SEA) with modal analysis, as for example the hybrid method proposed by Langley and Bremner [15].

A third class of methods uses specific reduction bases. Soize [16] has proposed an energy operator approach. An energy operator adapted to a fixed medium frequency band is defined and its dominant eigensubspace is used to construct a reduced model based on a Ritz-Galerkin method. Sarkar and Ghanem [17] have proposed the proper orthogonal decomposition method. The spatially dominant coherent structures in the vibration wave field in the medium frequency band are extracted from either numerical or physical data.

To estimate the prediction accuracy of a model, the uncertainties and modelling errors must be incorporated into the computational analysis. Ibrahim [18] and Manohar and Ibrahim [19] have given an extensive review of such approaches.

A first class of probabilistic methods falls in the framework of the stochastic finite element method (SFEM). Such methods only allow incorporating the parameter uncertainties into the analysis. The local parameters of the model (the material and geometrical properties) are described by random fields that are subsequently expanded or discretized. Ghanem and Spanos [20] have used the Karhunen-Loeve expansion. Such methods involve the inversion of a random system matrix. Ghanem and Spanos [20] have used an orthogonal series expansion for the random response field to obtain a set of algebraic equations.

A second class of probabilistic methods introduces the uncertainty directly on the modal properties of the total system or on the modal properties of possible subsystems. Mace and Shorter [21] have proposed a local modal/ perturbational method. Probability distributions are proposed for the modal properties of the subsystems and the resulting uncertainty on the modal properties of the total system is obtained with a perturbation relationship. A Monte Carlo simulation is elaborated to estimate the statistics of the frequency response functions.

A non-probabilistic approach has also been proposed. Chen et al. [22] have modelled the uncertain parameters through the specification of the lower and upper bounds of their values and have handled the subsequent problem of response analysis within the framework of interval algebra.

Soize [23,24] has proposed the non-parametric probabilistic approach. This approach builds a nonparametric probabilistic model associated with a deterministic reduced matrix model by substituting the reduced matrices of this model by fully populated random matrices. The probability distribution for the random matrices is derived from the entropy optimization principle [25,26]. The random matrices are modelled such that their mean values are the matrices of the deterministic prediction model, while ensuring that their generic characteristics, such as positive-definiteness, are preserved. A set of real valued dispersion parameters, one for each random matrix, allows controlling the dispersion of the probabilistic model. The first advantage of the approach is that it does not require the characterization of the local parameters of the model in terms of random fields or probability distributions. The second advantage is that it allows incorporating both the parameter uncertainties and the modelling errors into the analysis. Chebli [27] has combined the approach with the Craig-Bampton substructuring method.

The present paper follows the non-parametric probabilistic approach to estimate the prediction accuracy of dynamic soil-structure interaction models based on a coupled FE-BE formulation and on the Craig-Bampton substructuring method. It is shown how a non-parametric probabilistic model can be built associated with such a prediction model. The appropriate set of dispersion parameters is identified from an experimental data set. For this set of dispersion parameters, the dispersion of the random response predicted by the probabilistic model characterizes the level of uncertainty on this response and is used as an estimate of the prediction accuracy of the model.

The primary contribution of this paper is the application of the methodology to a case history. A prediction model is considered for the transmission of vibrations from the metro tunnel of the RER B line of RATP to the Maison du Mexique, a large six storey reinforced concrete frame structure, at the site of Cité Universitaire in Paris.

\section{Theoretical and numerical modelling}

This section first recalls how a deterministic dynamic soil-structure interaction model is built based on 
a three-dimensional coupled FE-BE formulation and on the Craig-Bampton substructuring method. Aubry and Clouteau [5] and Clouteau and Aubry [6] have given an extensive overview of this approach. It is shown how a non-parametric probabilistic model can be built associated with the deterministic model. The random eigenvalue problem is subsequently elaborated. Finally, a method is discussed to identify the dispersion parameters of the probabilistic model from experimental results.

\subsection{The deterministic dynamic soil-structure interaction model}

The dynamic soil-structure interaction problem is solved with a subdomain approach. The three-dimensional physical domain is decomposed into two subdomains: the soil domain, denoted by $\Omega_{s}$, and the structure domain, denoted by $\Omega_{b}$. These two subdomains are coupled throughout their common interface denoted by $\Sigma_{b s}$.

Let $\boldsymbol{u}_{\alpha}(\boldsymbol{x}, \omega)$ with $\alpha \in\{b, s\}$ be the dynamic perturbations of the static displacement fields in $\Omega_{\alpha}$ due to the dynamic loads. $\boldsymbol{x}$ is the position and $\omega$ is the circular frequency. The dynamic perturbations $\boldsymbol{\sigma}_{\alpha}\left(\boldsymbol{u}_{\alpha}\right)$ of the stress tensors are expressed as functions of the dynamic fluctuations $\boldsymbol{\varepsilon}\left(\boldsymbol{u}_{\alpha}\right)$ of the strain tensors

$\boldsymbol{\sigma}_{\alpha}\left(\boldsymbol{u}_{\alpha}\right)=\boldsymbol{C}_{\alpha} \varepsilon\left(\boldsymbol{u}_{\alpha}\right)$

$\boldsymbol{\varepsilon}\left(\boldsymbol{u}_{\alpha}\right)=\frac{1}{2}\left[\operatorname{grad} \boldsymbol{u}_{\alpha}+\left(\operatorname{grad} \boldsymbol{u}_{\alpha}\right)^{\mathrm{T}}\right]$

where $C_{b}$ is the fourth-order visco-elastic tensor:

$\boldsymbol{C}_{b}=\boldsymbol{A}_{b}-\mathrm{i} \omega \boldsymbol{B}_{b}$

The tensors $\boldsymbol{A}_{b}$ and $\boldsymbol{B}_{b}$ with the real elastic and the real damping coefficients verify the usual properties of symmetry and positiveness.

In the following, the traction vectors on an interface with an outer normal vector $\boldsymbol{n}$ are denoted by $\boldsymbol{t}_{\alpha}\left(\boldsymbol{u}_{\alpha}\right)$ :

$\boldsymbol{t}_{\alpha}\left(\boldsymbol{u}_{\alpha}\right)=\boldsymbol{\sigma}_{\alpha}\left(\boldsymbol{u}_{\alpha}\right) \boldsymbol{n}$

The equilibrium equations for the soil domain $\Omega_{s}$ and the boundary conditions on the free soil surface $\Gamma_{s \sigma}$ read as:

$\operatorname{div} \sigma_{s}\left(\boldsymbol{u}_{s}\right)+\boldsymbol{g}_{s}=-\rho_{s} \omega^{2} \boldsymbol{u}_{s}$ in $\Omega_{s}$

$\boldsymbol{t}_{s}\left(\boldsymbol{u}_{s}\right)=0 \quad$ on $\Gamma_{s \sigma}$

Radiation conditions are not needed since damping is accounted for. $\boldsymbol{g}_{s}(\boldsymbol{x}, \omega)$ is the vector of the body forces applied in the soil domain $\Omega_{s}$.

The balance of momentum for the structure domain $\Omega_{b}$ and the boundary conditions on the free structure boundary $\Gamma_{b \sigma}$ read as:

$\operatorname{div} \sigma_{b}\left(\boldsymbol{u}_{b}\right)=-\rho_{b} \omega^{2} \boldsymbol{u}_{b}$ in $\Omega_{b}$

$\boldsymbol{t}_{b}\left(\boldsymbol{u}_{b}\right)=0$ on $\Gamma_{b \sigma}$
Continuity of displacements and equilibrium of stresses hold on the soil-structure interface $\Sigma_{b s}$ :

$\boldsymbol{u}_{b}=\boldsymbol{u}_{s}$ on $\Sigma_{b s}$

$\boldsymbol{t}_{s}\left(\boldsymbol{u}_{s}\right)+\boldsymbol{t}_{b}\left(\boldsymbol{u}_{b}\right)=0$ on $\Sigma_{b s}$

The displacement field $\boldsymbol{u}_{s}(\boldsymbol{x}, \omega)$ is decomposed in the incident field $\boldsymbol{u}_{i}(\boldsymbol{x}, \omega)$, the locally diffracted field $\boldsymbol{u}_{d 0}(\boldsymbol{x}, \omega)$ and the field $\boldsymbol{u}_{s c}\left(\boldsymbol{u}_{b}\right)(\boldsymbol{x}, \omega)$ scattered by the structure into the soil

$\boldsymbol{u}_{s}=\boldsymbol{u}_{i}+\boldsymbol{u}_{d 0}+\boldsymbol{u}_{s c}\left(\boldsymbol{u}_{b}\right)$

such that these fields verify the following compatibility equations:

$\boldsymbol{u}_{i}+\boldsymbol{u}_{d 0}=0$ on $\Sigma_{b s}$

$\boldsymbol{u}_{s c}\left(\boldsymbol{u}_{b}\right)=\boldsymbol{u}_{b}$ on $\Sigma_{b s}$

The equilibrium of the structure is written in a weak variational form

$$
\begin{gathered}
\int_{\Omega_{b}} \varepsilon\left(\boldsymbol{v}_{b}\right): \sigma_{b}\left(\boldsymbol{u}_{b}\right) \mathrm{d} V-\omega^{2} \int_{\Omega_{b}} \rho_{b} \boldsymbol{v}_{b} \cdot \boldsymbol{u}_{b} \mathrm{~d} V \\
=\int_{\Sigma_{b s}} \boldsymbol{v}_{b} \cdot \boldsymbol{t}_{b}\left(\boldsymbol{u}_{b}\right) \mathrm{d} S
\end{gathered}
$$

with $v_{b}(\boldsymbol{x}, \omega)$ any virtual field defined in the structure domain $\Omega_{b}$.

Accounting for the stress equilibrium along the soilstructure interface $\Sigma_{b s}$, the weak variational formulation yields to:

$$
\begin{aligned}
& \int_{\Omega_{b}} \varepsilon\left(\boldsymbol{v}_{b}\right): \sigma_{b}\left(\boldsymbol{u}_{b}\right) \mathrm{d} V-\omega^{2} \int_{\Omega_{b}} \rho_{b} \boldsymbol{v}_{b} \cdot \boldsymbol{u}_{b} \mathrm{~d} V \\
& +\int_{\Sigma_{b s}} \boldsymbol{v}_{b} \cdot \boldsymbol{t}_{s}\left(\boldsymbol{u}_{s c}\left(\boldsymbol{u}_{b}\right)\right) \mathrm{d} S=-\int_{\Sigma_{b s}} \boldsymbol{v}_{b} \cdot \boldsymbol{t}_{s}\left(\boldsymbol{u}_{i}+\boldsymbol{u}_{d 0}\right) \mathrm{d} S
\end{aligned}
$$

The numerical solution of the dynamic soil-structure interaction problem is obtained based on the finite element method for the structure and the boundary element method for the soil.

The displacements $\boldsymbol{u}_{b}(\boldsymbol{x}, \omega)$ of the structure are discretized on a finite element basis:

$\boldsymbol{u}^{\mathrm{FE}}=\left[\begin{array}{c}\boldsymbol{u}_{0}^{\mathrm{FE}} \\ \boldsymbol{u}_{\Sigma}^{\mathrm{FE}}\end{array}\right]$

The vectors $\boldsymbol{u}_{0}^{\mathrm{FE}}$ and $\boldsymbol{u}_{\Sigma}^{\mathrm{FE}}$ contain the displacement field in the $n_{0}$ internal degrees of freedom of the structure and in the $n_{\Sigma}$ degrees of freedom of the soil-structure interface, respectively. The finite element stiffness, damping and mass matrices are denoted by $\underline{K}^{\mathrm{FE}}, \underline{C}^{\mathrm{FE}}$ and $\underline{M}^{\mathrm{FE}}$, respectively, and are block decomposed similarly to 
Eq. (16) as:

$\underline{A}^{\mathrm{FE}}=\left[\begin{array}{ll}\underline{A}_{00}^{\mathrm{FE}} & \underline{A}_{0 \Sigma}^{\mathrm{FE}} \\ \underline{A}_{\Sigma 0}^{\mathrm{FE}} & \underline{A}_{\Sigma \Sigma}^{\mathrm{FE}}\end{array}\right]$

with $\underline{A}^{\mathrm{FE}}=\underline{K}^{\mathrm{FE}}, \underline{C}^{\mathrm{FE}}$ or $\underline{M}^{\mathrm{FE}}$

The displacements $\boldsymbol{u}^{\mathrm{FE}}$ are subsequently expanded using the Craig-Bampton substructuring method

$\boldsymbol{u}^{\mathrm{FE}}=\left[\begin{array}{cc}\underline{\Phi}_{0} & \underline{\Phi}_{0}^{S} \\ 0 & \underline{\Phi}_{\Sigma}\end{array}\right]\left[\begin{array}{l}\boldsymbol{q}_{0} \\ \boldsymbol{q}_{\Sigma}\end{array}\right]=\underline{H} \boldsymbol{q}$

where $\underline{H}$ is the transformation matrix associated with the reduction basis and $\boldsymbol{q}$ is the vector of the generalized coordinates.

The displacements $\underline{\Phi}_{0}^{S}$ are the static transmissions of the $n_{s t}$ interface displacements $\underline{\Phi}_{\Sigma}$ into the structure (constraint modes), computed as

$\underline{\Phi}_{0}^{S}=-\left(\underline{K}_{00}^{\mathrm{FE}}\right)^{-1} \underline{K}_{0 \Sigma}^{\mathrm{FE}} \underline{\Phi}_{\Sigma}=\underline{S \Phi}_{\Sigma}$

where $\underline{S}$ is the finite element discretization of the elastostatic lifting operator.

The displacements $\underline{\Phi}_{0}$ are the $n_{c e}$ eigenmodes of the structure with vanishing displacements on the soil-structure interface (fixed interface eigenmodes) that verify

$\underline{K}_{00}^{\mathrm{FE}} \underline{\Phi}_{0}=\underline{\Omega}_{00}^{2} \underline{M}_{00}^{\mathrm{FE}} \underline{\Phi}_{0}$

where $\underline{\Omega}_{00}$ is the diagonal matrix containing the eigenfrequencies.

On this basis, the following deterministic reduced matrix model is obtained

$\left[\underline{K}_{b}-\mathrm{i} \omega \underline{C}_{b}-\omega^{2} \underline{M}_{b}+\underline{Z}_{s}(\omega)\right] \boldsymbol{q}(\omega)=\underline{\boldsymbol{f}}(\omega), \quad \omega \in B$

$\boldsymbol{u}^{\mathrm{FE}}(\omega)=\underline{H} \boldsymbol{q}(\omega)$

with

$\underline{K}_{b}=\underline{H}^{\mathrm{T}} \underline{K}^{\mathrm{FE}} \underline{H}=\left[\begin{array}{ll}\underline{K}_{00} & \underline{K}_{0 \Sigma} \\ \underline{K}_{0 \Sigma}^{\mathrm{T}} & \underline{K}_{\Sigma \Sigma}\end{array}\right]=\left[\begin{array}{cc}\underline{\Omega}_{00}^{2} & 0 \\ 0 & \underline{K}_{\Sigma \Sigma}\end{array}\right]$
$\underline{C}_{b}=\underline{H}^{\mathrm{T}} \underline{C}^{\mathrm{FE}} \underline{H}=\left[\begin{array}{ll}\underline{C}_{00} & \underline{C}_{0 \Sigma} \\ \underline{C}_{0 \Sigma}^{\mathrm{T}} & \underline{C}_{\Sigma \Sigma}\end{array}\right]$
$\underline{M}_{b}=\underline{H}^{\mathrm{T}} \underline{M}^{\mathrm{FE}} \underline{H}=\left[\begin{array}{ll}\underline{M}_{00} & \underline{M}_{0 \Sigma} \\ \underline{M}_{0 \Sigma}^{\mathrm{T}} & \underline{M}_{\Sigma \Sigma}\end{array}\right]=\left[\begin{array}{cc}I_{n_{s t}} & \underline{M}_{0 \Sigma} \\ \underline{M}_{0 \Sigma}^{\mathrm{T}} & \underline{M}_{\Sigma \Sigma}\end{array}\right]$

$\underline{Z}_{s}(\omega)=\left[\begin{array}{cc}0 & 0 \\ 0 & \underline{K}_{s}(\omega)\end{array}\right]$

$\underline{f}(\omega)=\left[\begin{array}{c}0 \\ \boldsymbol{f}_{s}(\omega)\end{array}\right]$ where $B$ is the frequency band of analysis and $I_{n_{s t}}$ is the identity matrix of dimension $n_{s t}$.

The matrices $\underline{K}_{b}$ and $\underline{C}_{b}$ are the positive semi-definite reduced stiffness and damping matrices of the structure and $\underline{M}_{b}$ is the positive-definite reduced mass matrix of the structure. The matrix $\underline{K}_{s}(\omega)$ is the dynamic soil impedance matrix and $f_{s}(\omega)$ is the vector of the generalized forces applied on the soil-structure interface $\Sigma_{b s}$, computed with a boundary element formulation for the soil domain.

\subsection{The associated non-parametric probabilistic model}

It is assumed that the influence of the parameter uncertainties and modelling errors related to the soil model and to the incident field can be neglected with respect to the influence of those related to the structure model. A non-parametric probabilistic model [23,24] associated with the deterministic model (21)-(22) is built by substituting the reduced matrices of the structure by fully populated random matrices. This construction allows to model the propagation of both the parameter uncertainties and the modelling errors related to the structure model to uncertainties on the model response since the dispersion of the random matrices may represent both uncertainty sources. The following non-parametric probabilistic model is obtained:

$\left[\boldsymbol{K}_{b}-\mathrm{i} \omega \boldsymbol{C}_{b}-\omega^{2} \boldsymbol{M}_{b}+\underline{Z}_{s}(\omega)\right] \boldsymbol{Q}(\omega)=\underline{\boldsymbol{f}}(\omega), \quad \omega \in B$

$\boldsymbol{U}^{\mathrm{FE}}(\omega)=\underline{H} Q(\omega)$

$\boldsymbol{K}_{b}, \boldsymbol{C}_{b}$ and $\boldsymbol{M}_{b}$ are the random reduced stiffness, damping and mass matrices of the building. $\boldsymbol{U}^{\mathrm{FE}}(\omega)$ and $\boldsymbol{Q}(\omega)$ are second-order stochastic processes indexed on $B$ with values in $\mathbb{C}^{n_{0}+n_{\Sigma}}$ and $\mathbb{C}^{n_{s t}+n_{c e}}$, respectively.

These random reduced matrices are normalized as follows:

$\boldsymbol{K}_{b}=\underline{S}_{K}^{\mathrm{T}} \boldsymbol{G}_{K} \underline{S}_{K} \quad$ with $\underline{K}_{b}=\underline{S}_{K}^{\mathrm{T}} \underline{S}_{K}$

$\boldsymbol{C}_{b}=\underline{S}_{C}^{\mathrm{T}} \boldsymbol{G}_{C} \underline{S}_{C} \quad$ with $\underline{C}_{b}=\underline{S}_{C}^{\mathrm{T}} \underline{S}_{C}$

$\boldsymbol{M}_{b}=\underline{L}_{M}^{\mathrm{T}} \boldsymbol{G}_{M} \underline{L}_{M} \quad$ with $\underline{M}_{b}=\underline{L}_{M}^{\mathrm{T}} \underline{L}_{M}$

$\boldsymbol{G}_{K}, \boldsymbol{G}_{C}$ and $\boldsymbol{G}_{M}$ are the positive-definite, normalized, random reduced stiffness, damping and mass matrix, respectively. $\underline{S}_{K}$ and $\underline{S}_{C}$ are obtained with a spectral decomposition of the positive semi-definite matrices $\underline{K}_{b}$ and $\underline{C}_{b} . \underline{L}_{M}$ is obtained with a Cholesky factorization of the positive-definite matrix $\underline{M}_{b}$.

The entropy optimization principle has been used by Soize [23,24] to construct the probabilistic model of the normalized random matrices $\boldsymbol{G}_{A}$, where $A$ is equal to $K, C$ or $M$, referring to the reduced stiffness, damping or mass matrix, respectively. The probability distribution of the 
normalized random matrix $\boldsymbol{G}_{A}$ maximizes entropy while satisfying the following constraints:

- $\boldsymbol{G}_{A}$ is a second-order random variable with values in the set of symmetric, positive-definite $n_{A} \times n_{A}$ real matrices.

- The mean value of $\boldsymbol{G}_{A}$ is the identity matrix $I_{n_{A}}$

$$
E\left\{\boldsymbol{G}_{A}\right\}=I_{n_{A}}
$$

where $E\{\cdot\}$ is the mathematical expectation.

- The inverse of $\boldsymbol{G}_{A}$ has a finite moment of order $\gamma_{A}$ with $\gamma_{A}$ a positive integer

$$
E\left\{\left\|\boldsymbol{G}_{A}^{-1}\right\|_{F}^{\gamma A}\right\}<+\infty
$$

with $\|\cdot\|_{F}$ the Frobenius norm.

Soize $[23,24]$ has obtained an analytical form for the resulting probability distribution of the normalized random matrix $\boldsymbol{G}_{A}$, depending on a single positive real valued dispersion parameter $\delta_{A}$, defined as:

$\delta_{A}^{2}=\frac{E\left\{\left\|\boldsymbol{G}_{A}-\underline{G}_{A}\right\|_{F}^{2}\right\}}{\left\|\underline{G}_{A}\right\|_{F}^{2}}$

The dispersion parameter $\delta_{A}$ is linked to the parameter $\gamma_{A}$ in the constraint (34) and to the dimension $n_{A}$. For $\gamma_{A}=2$, the constraint (34) is fulfilled if $\delta_{A}$ satisfies the following inequality:

$0 \leq \delta_{A}<\sqrt{\frac{n_{A}+1}{n_{A}+5}}<1$

The complete probabilistic model for the random reduced matrices $\boldsymbol{K}_{b}, \boldsymbol{C}_{b}$ and $\boldsymbol{M}_{b}$ is obtained from the probabilistic models for the normalized random matrices $\boldsymbol{G}_{K}, \boldsymbol{G}_{C}$ and $\boldsymbol{G}_{M}$ and is parameterized by the set of dispersion parameters $\delta_{K}, \delta_{C}$ and $\delta_{M}$. If each dispersion parameter satisfies inequality (36), it can be proved that the structure response $\boldsymbol{U}^{\mathrm{FE}}(\omega)$ is a second-order stochastic process.

In $[23,24]$, a direct Monte Carlo simulation procedure has been elaborated such that a set of realizations of the random matrices can be generated. Once the computation of the associated set of realizations of the random response is performed, statistical estimates of the response can be computed.

\subsection{The random eigenvalue problem}

In this section, the random eigenvalue problem defined by the non-parametric probabilistic model is elaborated.

The random reduced stiffness and mass matrices define the following random eigenvalue problem

$\boldsymbol{K}_{00} \boldsymbol{Q}_{\Phi}=\boldsymbol{\Omega}_{00}^{2} \boldsymbol{M}_{00} \boldsymbol{Q}_{\Phi}$

where $\boldsymbol{K}_{00}$ and $\boldsymbol{M}_{00}$ are random block submatrices of the random reduced stiffness matrix $\boldsymbol{K}_{b}$ and mass matrix $\boldsymbol{M}_{b}$, respectively. The matrix $\boldsymbol{Q}_{\Phi}$ contains the $n_{c e}$ random eigenvectors and $\boldsymbol{\Omega}_{00}$ is the random diagonal matrix containing the random eigenfrequencies.

The $n_{c e}$ random eigenvectors in the matrix $\boldsymbol{Q}_{\Phi}$ are considered as the random coordinates of a set of $n_{c e}$ random eigenmodes $\boldsymbol{\Phi}_{0}$ with respect to the deterministic eigenmodes $\underline{\Phi}_{0}$ :

$\boldsymbol{\Phi}_{0}=\underline{\Phi}_{0} \boldsymbol{Q}_{\Phi}$

The study of the random eigenmodes $\boldsymbol{\Phi}_{0}$ and of the random eigenfrequencies in $\boldsymbol{\Omega}_{00}$ permits investigating the sensitivity of the modal basis to small uncertainties on the mass or stiffness of the structure. The modes $\boldsymbol{\Phi}_{0}$ obtained by the resolution of the random eigenvalue problem in the space spanned by the deterministic eigenmodes are pertinent since it is known that small perturbations of the mass or stiffness of a structure modify the individual eigenvectors, but leave the space spanned by the eigenmodes unchanged [9-11].

\subsection{Confidence regions associated with given probability levels}

The dispersion of the random response predicted by the probabilistic model is characterized by estimating appropriate confidence regions. The size of the confidence regions represents the dispersion of the random response. For the $k$ th degree of freedom $X_{k}(\omega)$ of the random response $\boldsymbol{X}(\omega)$ at the circular frequency $\omega$, a confidence region is built on a logarithmic scale associated with a given probability level $P_{c}$ :

$p\left(d B_{k}^{-}(\omega)<d B_{k}(\omega)<d B_{k}^{+}(\omega)\right) \geq P_{c}$

$d B_{k}(\omega)=20 \log _{10}\left(\left|X_{k}(\omega)\right| / X^{\mathrm{ref}}\right)$

$X^{\text {ref }}$ is a reference value equal to $1 \mathrm{~m} /(\mathrm{N} \mathrm{Hz})$ if $\boldsymbol{X}(\omega)$ is a compliance and equal to $1 \mathrm{~m} / \mathrm{N}$ if $\boldsymbol{X}(\omega)$ is a mobility. The lower and upper bounds $d B_{k}^{-}(\omega)$ and $d B_{k}^{+}(\omega)$ are built using the Chebychev inequality based on statistical estimates of the mean value and of the variance of $X_{k}(\omega)$ [27].

\subsection{Identification of the dispersion parameters from experimental results}

The dispersion of the random reduced matrices $\boldsymbol{K}_{b}, \boldsymbol{C}_{b}$ and $\boldsymbol{M}_{b}$ is controlled by the set of positive real valued dispersion parameters $\delta_{K}, \delta_{C}$ and $\delta_{M}$.

The dispersion of the random reduced matrices $\boldsymbol{K}_{b}, \boldsymbol{C}_{b}$ and $\boldsymbol{M}_{b}$ of the probabilistic model must represent the level of uncertainty on the reduced stiffness, damping and mass matrix of the model, respectively, due to the parameter uncertainties and the modelling errors present in the model. In this case, the dispersion of the random response of the probabilistic model represents the uncertainty on the model response due to these parameter uncertainties and modelling 
errors (it is assumed that the relation between the reduced matrices and the model response is correct).

In the present paper, the dispersion parameters are identified from an experimental data set. They are tuned such that the confidence regions for the random response predicted by the probabilistic model envelope best estimates for the response of the structure derived from experimental results. For this set of tuned dispersion parameters, the dispersion of the random response, represented by the size of the associated confidence regions, is used as an estimate of the prediction accuracy of the model.

\section{Outline of the case history: the Cité Universitaire site}

A prediction model for the transmission of vibrations from a metro tunnel to a nearby building is considered. It is assumed that the interaction of the track, the tunnel and the soil is weakly coupled to the interaction of the soil and the building. Based on this assumption, the transmission of the vibrations is modelled with two submodels. First, an interaction model of the track, the tunnel and the soil is used to compute the wave field radiated by the tunnel into the soil. Next, an interaction model of the soil and the building is used to compute the structural vibration induced by this wave field. Furthermore, it is assumed that the influence of the parameter uncertainties and modelling errors related to the track, the tunnel and the soil can be neglected with respect to those related to the building. The track-tunnelsoil interaction model is kept deterministic and, in order to estimate the prediction accuracy of the complete model, a non-parametric probabilistic model is constructed associated with the soil-building interaction model.

Results are presented for the Cité Universitaire site in Paris, located on the RER B line of RATP between the metro stations Cité Universitaire and Gentilly. Fig. 1(a) shows a schematic view of the site. The tunnel is a masonry cut-and-cover tunnel at a shallow depth of about $9.3 \mathrm{~m}$ below the free soil surface embedded in sand layers. Two classic ballast tracks are running in the tunnel. The rails are supported by grooved rubber pads and are resting on monoblock concrete sleepers. For more information on the tunnel and track characteristics, the reader is referred to Clouteau et al. [3].

A spectral analysis of surface waves (SASW) test has been performed in order to determine the thickness and the dynamic characteristics of the shallow soil layers [28]. The tests have been performed on two measurement lines, one line perpendicular to the tunnel and one line parallel to the tunnel, and demonstrate the presence of a thin layer with a thickness of approximately $1.4 \mathrm{~m}$ and a shear wave velocity of $C_{s}=115 \mathrm{~m} / \mathrm{s}$, a stiffer layer with a thickness of $3.0 \mathrm{~m}$ and a shear wave velocity of $C_{s}=220 \mathrm{~m} / \mathrm{s}$ on top of a half-space with a shear wave velocity $C_{s}=315 \mathrm{~m} / \mathrm{s}$.

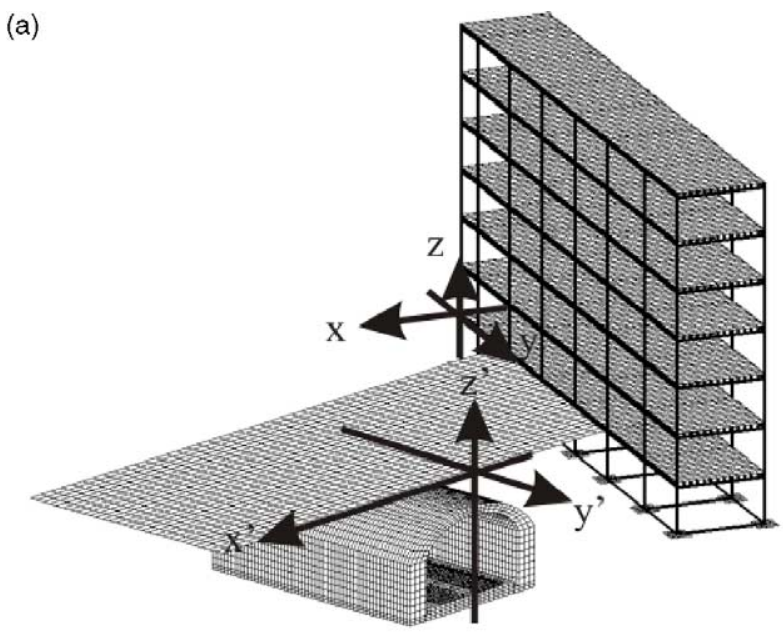

(b)

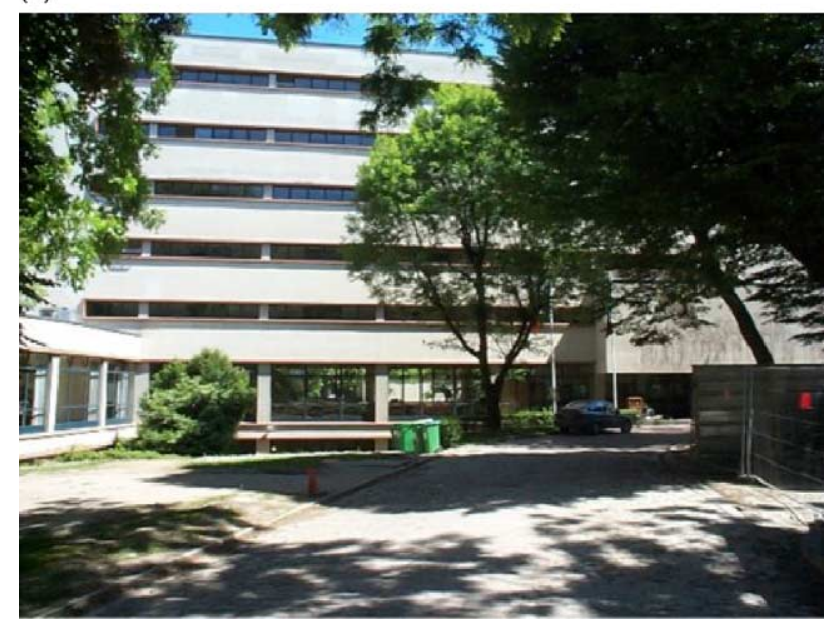

Fig. 1. (a) Schematic view of the Cité Universitaire site and (b) side view of the Maison du Mexique.

The transmission of vibrations into the Maison du Mexique, a six storey student dormitory, is studied. Fig. 1(b) shows a side view of the building. The main building is a reinforced concrete frame structure. It has two sets of eight columns such that the floor spans are approximately $6.2 \mathrm{~m}$. The floor-to-ceiling height is approximately $2.85 \mathrm{~m}$. Very few information is available on the foundation of the building. Therefore, assumptions have been made regarding the foundation included in the numerical model, which consists of an independent foundation plate under each column. The dimensions of these plates have been determined from estimates of the required bearing capacity. For more information on the building characteristics, the reader is referred to Chatterjee et al. [29], Coster [30], and Arnst [31].

For the tunnel, a right-handed Cartesian frame of reference $\left(x^{\prime}, y^{\prime}, z^{\prime}\right)$ is defined with the origin at the free soil surface (Fig. 1(a)). For the building, a right-handed Cartesian frame of reference $(x, y, z)$ is defined with the origin in the upper left corner on the ground floor (Fig. 1(b)). The origin of the reference frame of the building has 
coordinates $\left(x^{\prime}=23.5 \mathrm{~m}, y^{\prime}=-24.3 \mathrm{~m}, z^{\prime}=0 \mathrm{~m}\right)$ in the reference frame of the tunnel. The angle between the $y$-and the $y^{\prime}$-axis is $25^{\circ}$.

\section{In situ measurements at the Cité Universitaire site}

Measurements have been performed for site characterization as well as to obtain data for the estimation of the prediction accuracy of the model [29]. Accelerometers have been placed at different locations in the Maison du Mexique (Fig. 2). Vibrations have been generated by an impact of an instrumented hammer with a mass of $5.3 \mathrm{~kg}$ and a soft tip on the rail head in the point with coordinates $\left(x^{\prime}=-2.5 \mathrm{~m}\right.$, $\left.y^{\prime}=0 \mathrm{~m}, z^{\prime}=-8.2 \mathrm{~m}\right)$.

In the present paper, only the vertical response at measurement locations close to the excitation point is considered: the experimental degrees of freedom BA01z in the basement near column C1, F0PLz at mid-span of the slab on the ground floor between columns $\mathrm{C} 2$ and $\mathrm{C} 3$ and $\mathrm{F} 201 \mathrm{z}$ at the edge of the slab on the second floor near column $\mathrm{C} 1$ (Fig. 2). The location BA01 has coordinates $(x=-0.5 \mathrm{~m}, y=26.5 \mathrm{~m}, z=-3.6 \mathrm{~m})$, FOPL has coordinates $(x=-2.9 \mathrm{~m}, y=18.2 \mathrm{~m}, z=0 \mathrm{~m})$ and F201 has coordinates $(x=-0.5 \mathrm{~m}, y=26.5 \mathrm{~m}, z=6.8 \mathrm{~m})$.

A total of 25 events have been recorded. From this set of events, best estimates have been extracted for the frequency response functions (FRFs) using the $\hat{H}_{1}(f)$ estimator [32]. The FRF is defined as the ratio of the response to the force applied on the rail head. The particular term mobility is used in this paper when the ratio of the velocity of the response to the applied force is considered. The coherence functions between the applied force and the measured responses have also been estimated, as well as confidence regions for the measured FRFs. Figs. 3 and 4 show the time history during the sixth event, the best estimate for the FRF, the confidence region for the measured FRFs and the coherence function for the experimental DOFs BA01z and FOPLz, respectively.

Due to noise, low coherence values are observed at low frequencies below $20 \mathrm{~Hz}$ and at high frequencies above

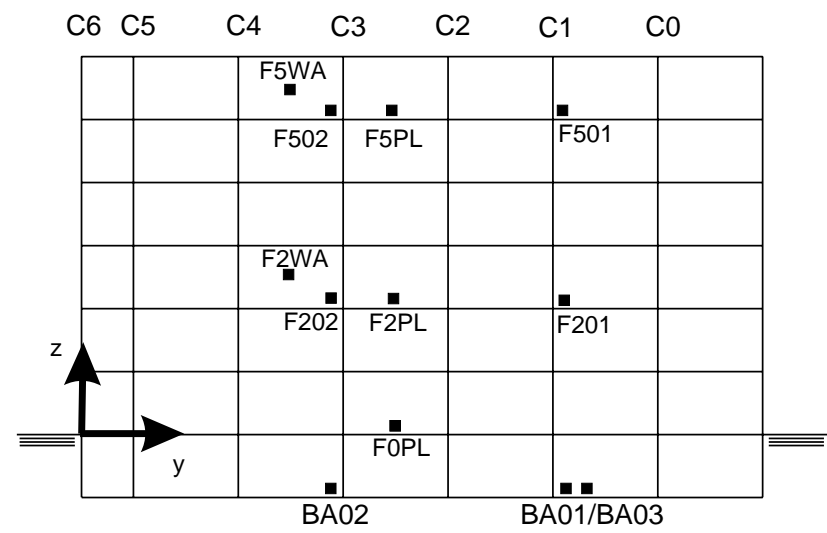

Fig. 2. The measurement locations in the Maison du Mexique.
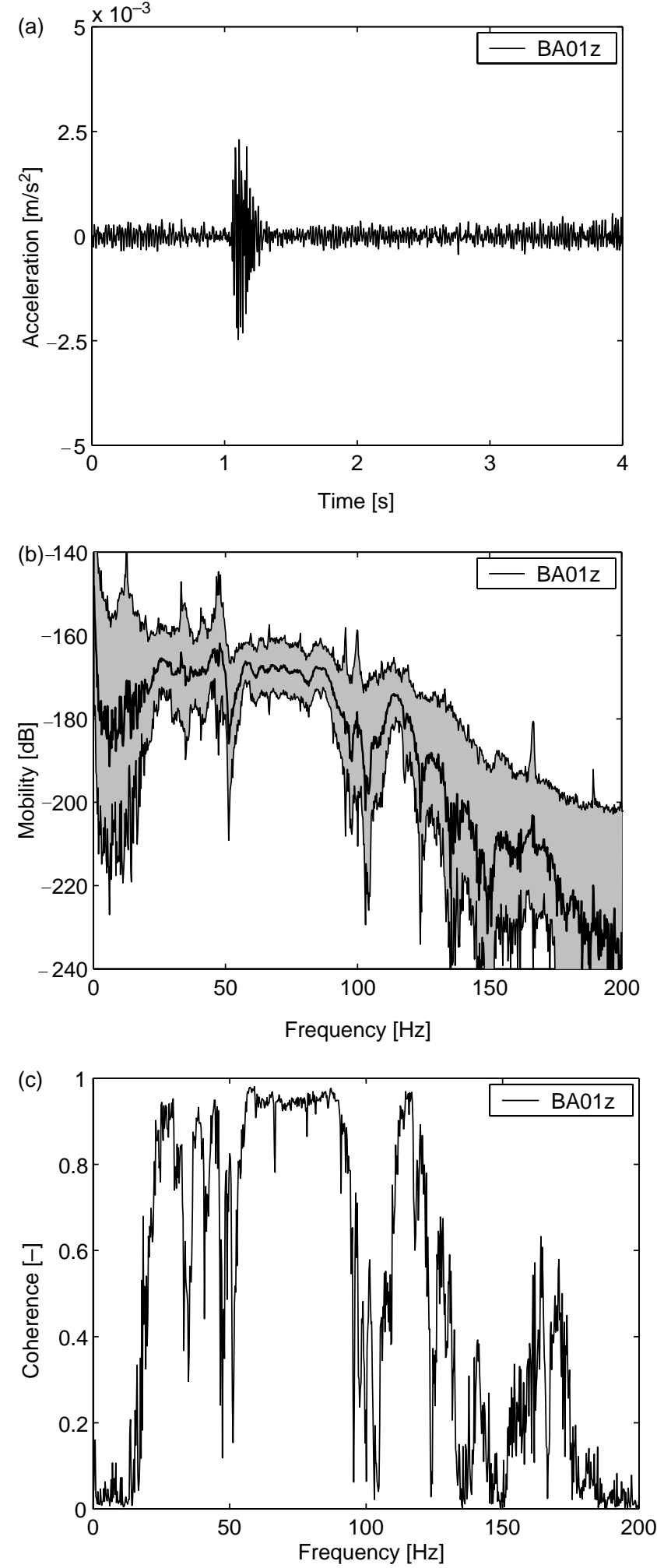

Fig. 3. Vertical response in the basement in the DOF BA01z: (a) time history during the sixth event, (b) amplitude of the best estimate of the FRF (mobility) (thick solid line) and confidence region $\left(P_{c}=0.95\right)$ for the measured FRFs (grey region) and (c) coherence function between the applied force and the measured response. 

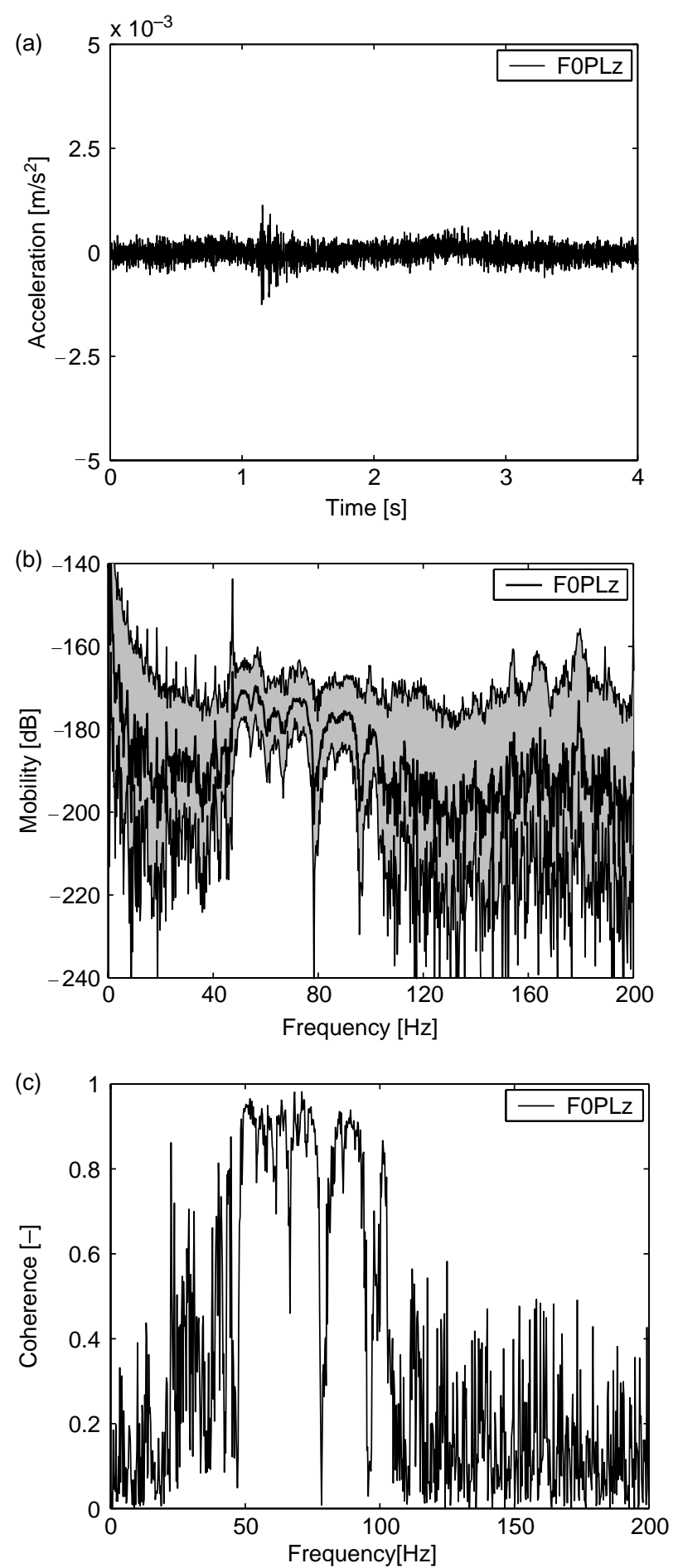

Fig. 4. Vertical response at the ground floor in the DOF FOPLz: (a) time history during the sixth event, (b) amplitude of the best estimate of the FRF (mobility) (thick solid line) and confidence region $\left(P_{c}=0.95\right)$ for the measured FRFs (grey region) and (c) coherence function between the applied force and the measured response.

$100 \mathrm{~Hz}$. The coherence values decrease with the distance to the impact point. Low values of the coherence function indicate a low quality of the data and correspond to a high level of uncertainty on the experimental results, as can be concluded from the size of the confidence regions.

\section{The deterministic model for the Cité Universitaire site}

In this section, the deterministic prediction model is elaborated to compute the FRFs from a vertical fixed point force on the rail head to the response in the building. This force is applied at the same point where the experimental impact took place. The model proposed by Clouteau et al. [3] based on a periodic coupled FE$\mathrm{BE}$ formulation is used to model the dynamic tracktunnel-soil interaction. The deterministic soil-structure interaction model (21)-(22) is subsequently elaborated to compute the structural response. The model is designed for computations in the frequency range between 0 and $100 \mathrm{~Hz}$. The SDT Toolbox [33] for Matlab is used for the finite element computations, while the software MISS3D [5] is used for the boundary element computations.

\subsection{The deterministic dynamic soil-structure interaction model}

The finite element model for the Maison du Mexique has been restricted to the main structural parts [30,31]. Only the columns, beams and floors of the main, reinforced concrete frame structure are included in the model. Two node beam elements are used for the columns and the beams. Four node quadrilateral plate elements are used for the floor slabs.

The soil-building interface $\Sigma_{b s}$ is restricted to the 16 foundation plates of the building that are assumed to be rigid and massless. The displacements on $\Sigma_{b s}$ are decomposed on a basis of interface modes that are the 96 rigid body modes of the 16 plates.

The basis of constraint modes is extended with fixed interface eigenmodes of the building. Fig. 5 shows the eigenfrequency and the eigenfrequency separation of the first 1500 eigenmodes. Fig. 6 shows the 2nd, the 35th and the 535th eigenmode. In the frequency range between 0 and $12 \mathrm{~Hz}$, global eigenmodes of longitudinal bending (1-4th), of transverse bending (1-3th) and of torsion (1-3th) are found. From $12 \mathrm{~Hz}$ to approximately $17 \mathrm{~Hz}$, a high density of local first-order plate bending modes is obtained, as well as some higher order global modes. From approximately $28 \mathrm{~Hz}$, a high density of local higher order plate bending modes is found with some high order global modes. The eigenfrequency of the 1500th eigenmode is $179.26 \mathrm{~Hz}$. The average eigenfrequency separation is $0.118 \mathrm{~Hz}$.

The damping of the structure is taken into account by a simplified model of the reduced damping matrix (24)

$\underline{C}_{b}=\left[\begin{array}{cc}\underline{C}_{00} & 0 \\ 0 & 0\end{array}\right]$ 

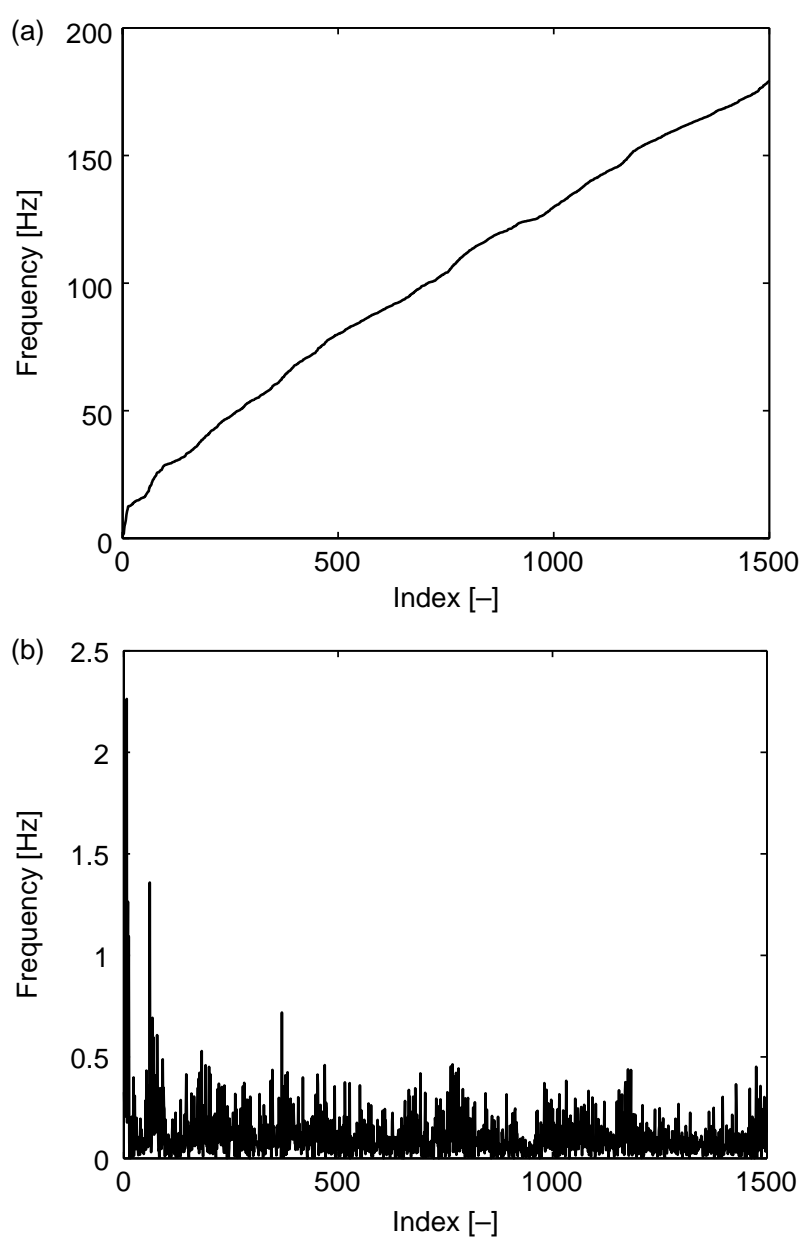

Fig. 5. (a) Eigenfrequency and (b) eigenfrequency separation of the first 1500 deterministic eigenmodes of the building. where $\underline{C}_{00}$ is a diagonal matrix with elements $2 \xi \underline{\omega}_{j}$ with $\underline{\omega}_{j}$ the pulsation of the $j$ th eigenmode and $\xi=$ 0.02 the modal damping ratio.

The discrete excitation frequencies are chosen based on the modal bandwidths. The frequency ranges from 0.25 to $15 \mathrm{~Hz}$, from 15.5 to $70 \mathrm{~Hz}$ and from 71 to $100 \mathrm{~Hz}$ are distinguished, wherein the frequency steps $0.25,0.5$ and $1 \mathrm{~Hz}$, respectively, are employed.

The convergence of the prediction model with respect to the number of eigenmodes is investigated by computing the FRFs in several degrees of freedom for different numbers of eigenmodes, as shown in Figs. 7 and 8 for the degrees of freedom BA01z and F0PLz. A number of 1000 eigenmodes is sufficient to reach a reasonable convergence of the deterministic model response. In the following, the computations are performed with 1500 eigenmodes.

\subsection{The deterministic building response}

Fig. 9 shows the harmonic response of the tunnel, the soil and the building due to a fixed harmonic load on the rail head at the frequencies 15 and $60 \mathrm{~Hz}$. The low frequency response is mainly governed by global low order bending and torsion of the whole building and by first-order local plate bending of the slabs (Fig. 9(a)). At higher frequencies, the response is mainly governed by local plate bending of the slabs and also by higher order bending and torsion of the whole building (Fig. 9(b)).

Figs. 10 and 11 show the FRFs from the force on the rail head to the response in three points near column $C 1$ in the basement, at the ground floor and at the second floor. At low frequencies, the amplitude of the FRFs shows clear peaks that correspond to the resonances of low frequency global eigenmodes. The sharpness of the peaks decreases with the (a)

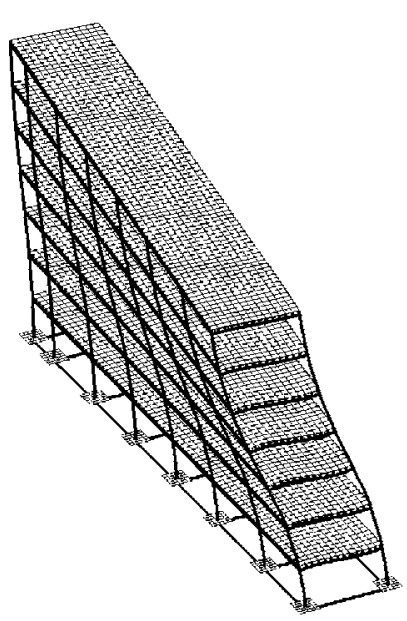

(b)

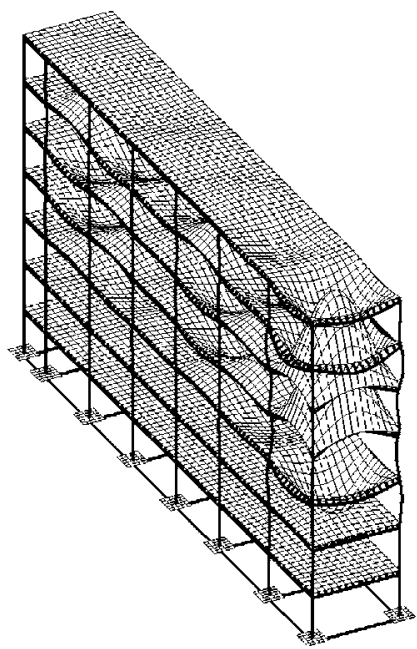

(c)

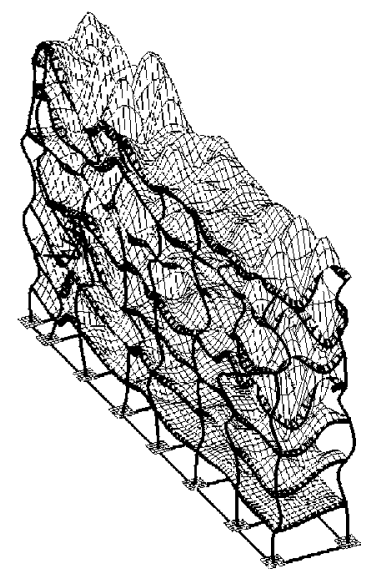

Fig. 6. Deterministic (a) first-order global torsion-transverse bending mode, (b) first-order local plate bending mode and (c) higher order local plate bending mode. 

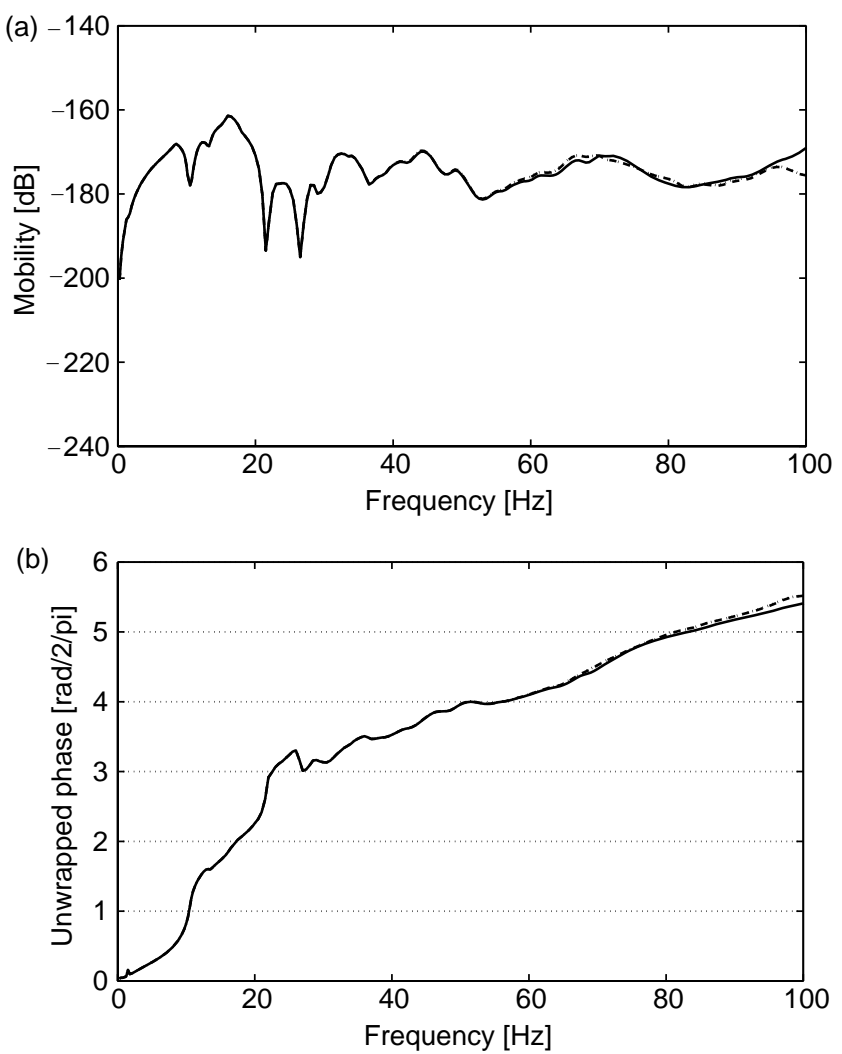

Fig. 7. (a) Amplitude and (b) unwrapped phase of the deterministic FRF (mobility) in the degree of freedom BA01z computed with 500 (solid line), 1000 (dashed line) and 1500 (dotted line) eigenmodes.

frequency. At higher frequencies, the amplitude of the horizontal component of the response is much smaller than the amplitude of the vertical component. Due to wave propagation, the phase increases with the frequency. Phase variations are less sharp as the frequency increases. Both the amplitude and the phase are important characteristics of the vibration in this frequency range.

\section{The non-parametric probabilistic model for the Cité Universitaire site}

\subsection{The probabilistic soil-structure interaction model}

The non-parametric probabilistic model associated with the deterministic soil-building interaction model is subsequently elaborated. For the sake of simplicity, as well as to avoid the expensive tuning of three dispersion parameters, it is assumed that the dispersion levels of the random reduced stiffness, damping and mass matrix are equal $\left(\delta_{K}=\delta_{C}=\delta_{M}=\delta\right)$.

\subsection{The random eigenvalue problem and convergence with respect to the dimension of the reduction basis}

The random eigenvalue problem presented in Section 2.3 has been elaborated for three different dimensions of
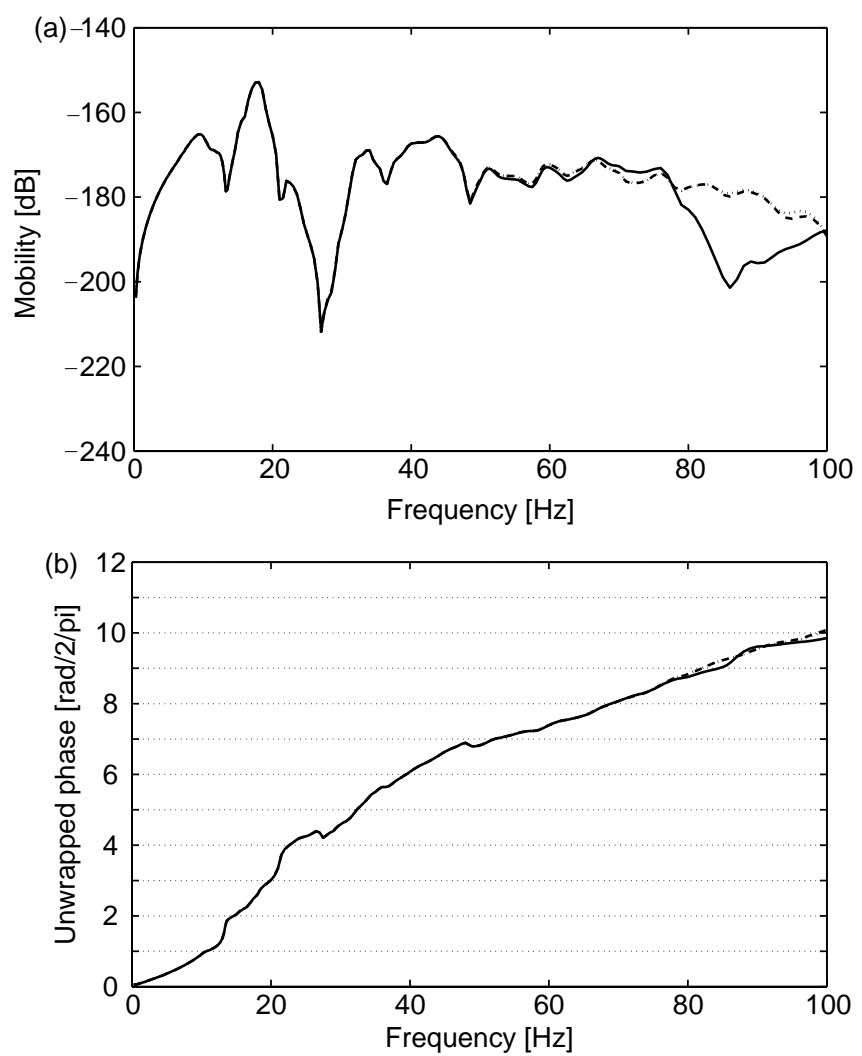

Fig. 8. (a) Amplitude and (b) unwrapped phase of the deterministic FRF (mobility) in the degree of freedom F0PLz computed with 500 (solid line), 1000 (dashed line) and 1500 (dotted line) eigenmodes.

the reduction basis of the soil-structure interaction model, namely for 1000,1500 , and 2000 eigenmodes included in this basis.

Fig. 12 shows the first 2000 deterministic eigenfrequencies and estimates of the mean values of the 1000,1500, and 2000 random eigenfrequencies, respectively, for a dispersion level $\delta=0.4$. Due to the non-linearity of the eigenvalue problem, the mean values of the random eigenfrequencies differ from the deterministic values. At low eigenfrequencies, the mean values of the random eigenvalues are generally smaller than the deterministic values. At the highest eigenfrequencies of a fixed reduction basis, the mean values of the random eigenfrequencies are significantly larger than the deterministic values. In the relevant eigenfrequency range (up to approximately 1.5 times the highest excitation frequency), a reasonable convergence of the mean values of the random eigenfrequencies with respect to the dimension of the reduction basis is obtained for 1500 eigenmodes.

Fig. 13 shows estimates of the standard deviations of the 1000,1500 , and 2000 random eigenfrequencies, respectively, for the same dispersion level $\delta=0.4$. The standard deviation generally increases with the eigenfrequency, illustrating that the sensitivity of eigenfrequencies to uncertainties on the mass and stiffness increases with the frequency. At the highest eigenfrequencies of a fixed 
(a)

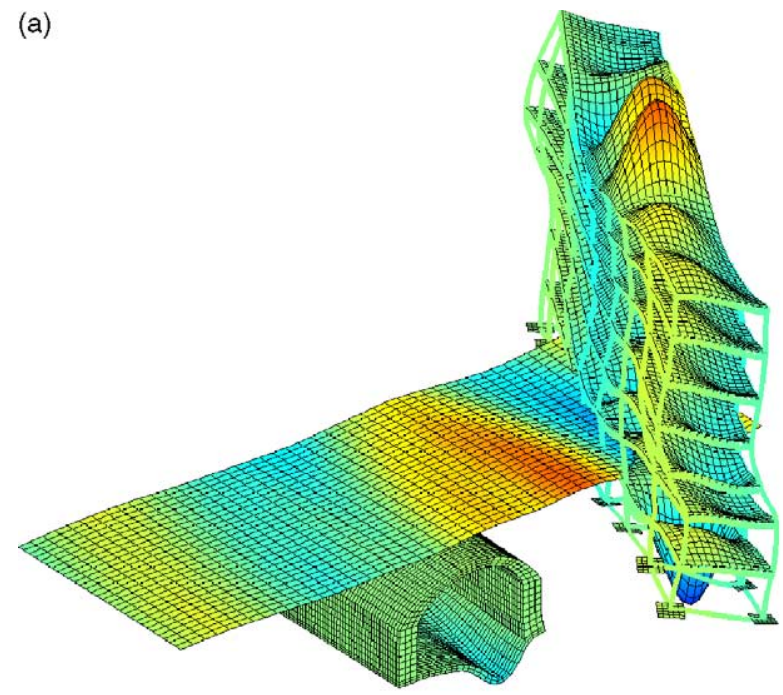

(b)

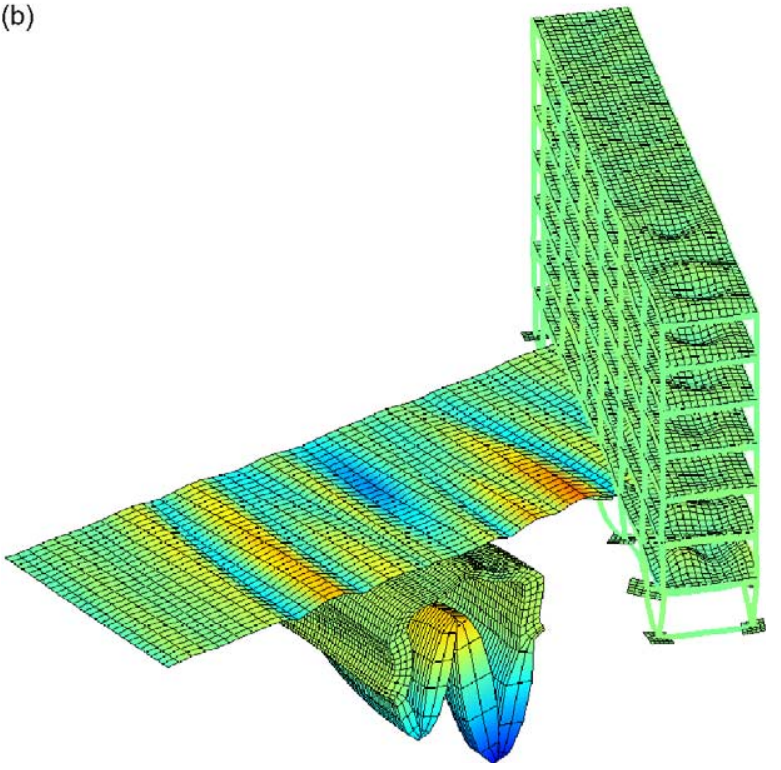

Fig. 9. Deterministic harmonic response of the tunnel, the free soil surface and the building at (a) $15 \mathrm{~Hz}$ and (b) $60 \mathrm{~Hz}$.

reduction basis, the standard deviation increases strongly. Reasonable convergence in the relevant eigenfrequency range is obtained for 1500 eigenmodes.

The previous results are important for the convergence of the random response with respect to the dimension of the reduction basis, as this convergence is linked to the convergence of the random eigenfrequencies in the relevant eigenfrequency range. It has been demonstrated that the convergence of the latter is only obtained when a reduction basis is used with eigenmodes with eigenfrequencies larger than the highest frequency of this eigenfrequency range. Generally, in order to attain convergence of the random response predicted by the non-parametric probabilistic model, a reduction basis must be used that is larger than the reduction basis required to attain convergence of the deterministic model.
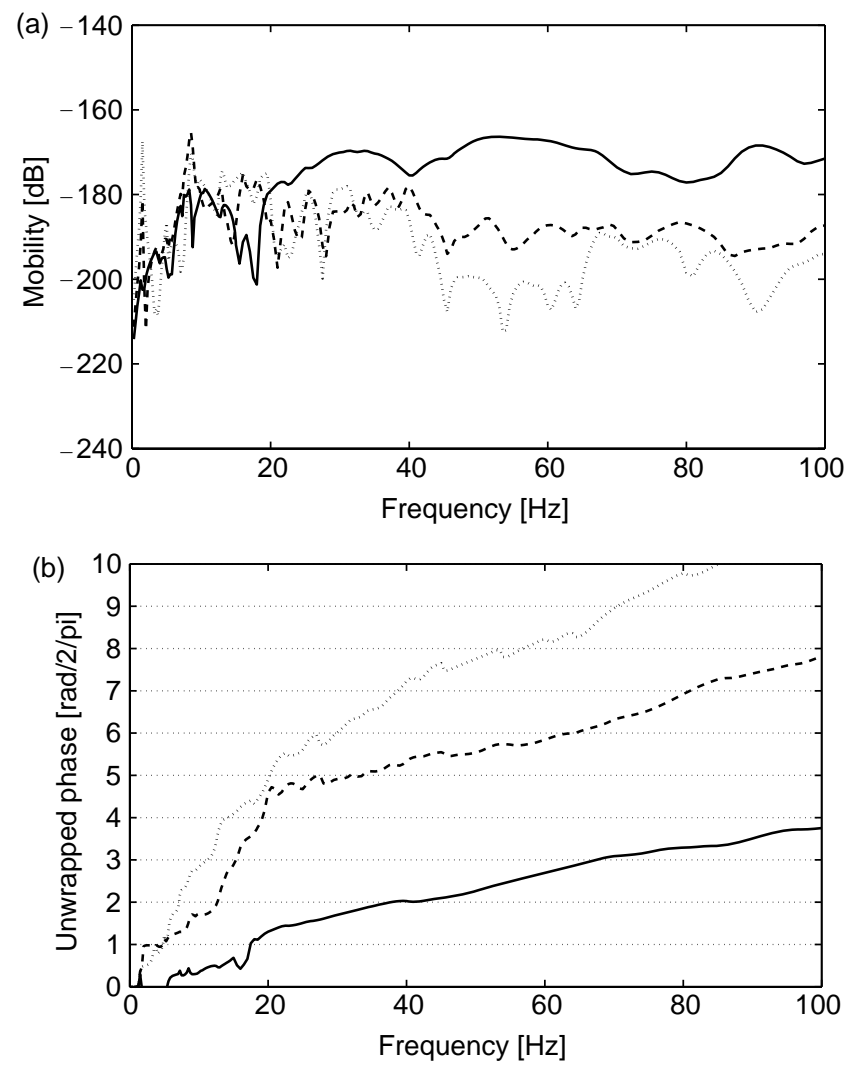

Fig. 10. (a) Amplitude and (b) unwrapped phase of the horizontal component of the deterministic FRFs (mobility) in the point BA01 (solid line), in the corresponding point at the ground floor $(x=-0.5 \mathrm{~m}, y=$ $26.5 \mathrm{~m}, z=0 \mathrm{~m}$ ) (dashed line) and at the second floor (F201) (dotted line).

In the following, all computations with the probabilistic model are performed with 1500 eigenmodes.

Figs. 14 and 15 show the first 50 coordinates of two realizations of the third (global first-order torsion) and 28th (local first-order plate bending) random eigenmodes when projected on the basis of deterministic eigenmodes for a dispersion level $\delta=0.4$. At low eigenfrequencies, where global bending and torsion modes of the building are found and the eigenfrequency separation is relatively large, the individual global eigenmodes are not modified by small perturbations of the mass and stiffness. At higher eigenfrequencies, where densely packed clusters of local eigenmodes are found, small perturbations of the mass and stiffness significantly modify the local eigenmodes.

\subsection{Convergence with respect to the number of Monte Carlo simulations}

The convergence of the estimates of the confidence regions for the random FRFs predicted by the probabilistic model with respect to the number of Monte Carlo simulations is analyzed through the convergence analysis of the statistics of the realizations of these random FRFs. 

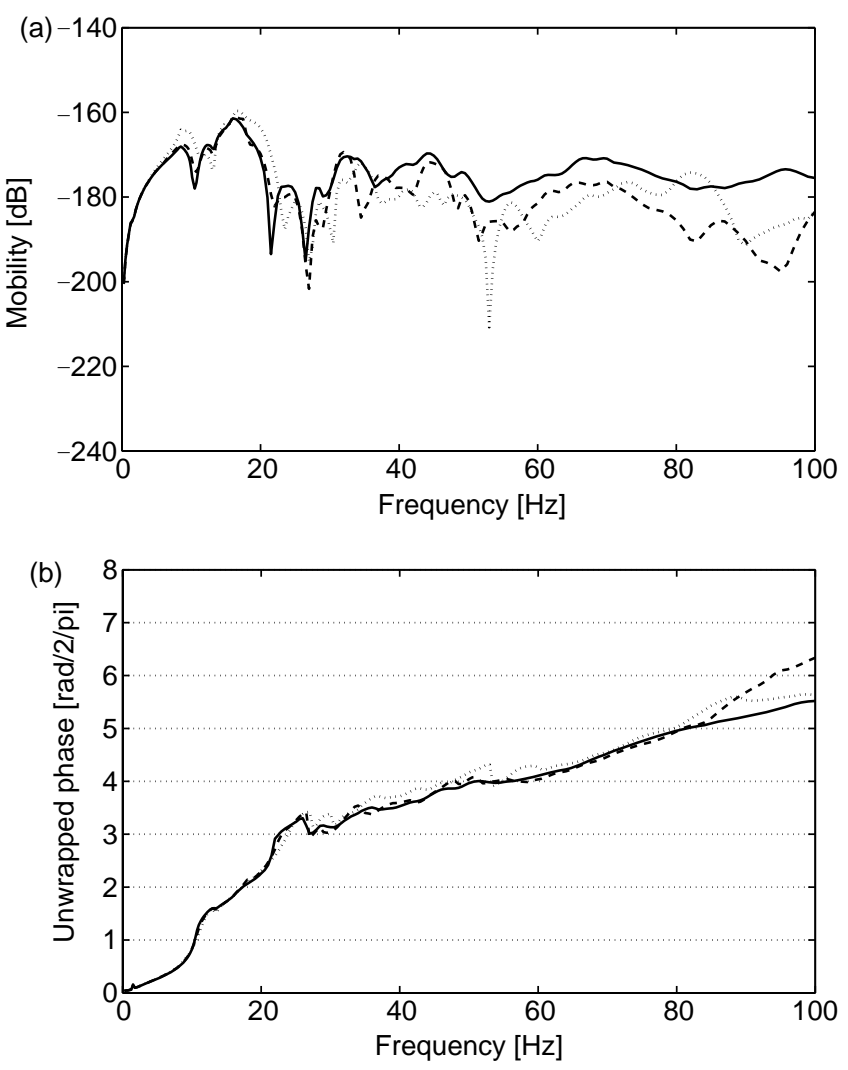

Fig. 11. (a) Amplitude and (b) unwrapped phase of the vertical component of the deterministic FRFs (mobility) in the point BA01 (solid line), in the corresponding point at the ground floor $(x=-0.5 \mathrm{~m}, y=26.5 \mathrm{~m}, z=0 \mathrm{~m})$ (dashed line) and at the second floor (F201) (dotted line).

Figs. 16 and 17 show the estimated mean value and standard deviation of the random FRF in the degree of freedom FOPLz as a function of the number of Monte Carlo simulations for different values of the dispersion level $\delta$ at the frequencies 50 and $100 \mathrm{~Hz}$, respectively. The larger the dispersion level $\delta$ and the higher the frequency, the larger the required number of simulations becomes. In the

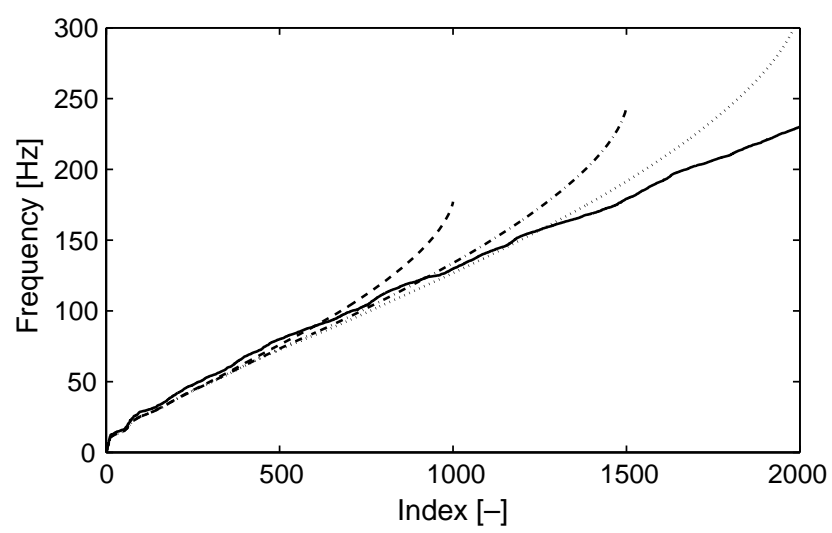

Fig. 12. The deterministic eigenfrequencies (solid line) and the estimated mean values of the random eigenfrequencies for $\delta=0.4$ computed with 1000 (dashed line), 1500 (dash-dotted line) and 2000 (dotted line) eigenmodes.

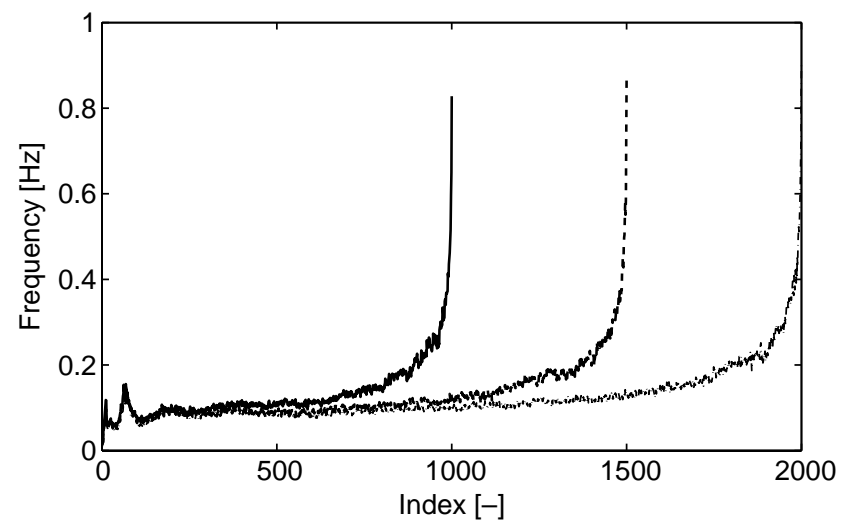

Fig. 13. Estimated standard deviations of the random eigenfrequencies for $\delta=0.4$ computed with 1000 (solid line), 1500 (dash-dotted line) and 2000 (dotted line) eigenmodes.

considered frequency range and for $\delta \leq 0.4,160$ Monte Carlo simulations seem to be sufficient to reach convergence. In the following, all computations are performed with 200 simulations.

\subsection{The random building response}

In this section, the sensitivity of the model response to arbitrary random uncertainties is studied. The dispersion of the random response is studied as a function of the dispersion of the random matrices.

Fig. 18 shows the estimated bounds of the confidence regions for the random FRFs in the degrees of freedom BA01z, FOPLz and F201z for different values of the dispersion level $\delta$. The size of the confidence regions, or the dispersion of the random response, increases with the dispersion level $\delta$.

The dispersion of the random response increases with the frequency. At very low frequencies, where global
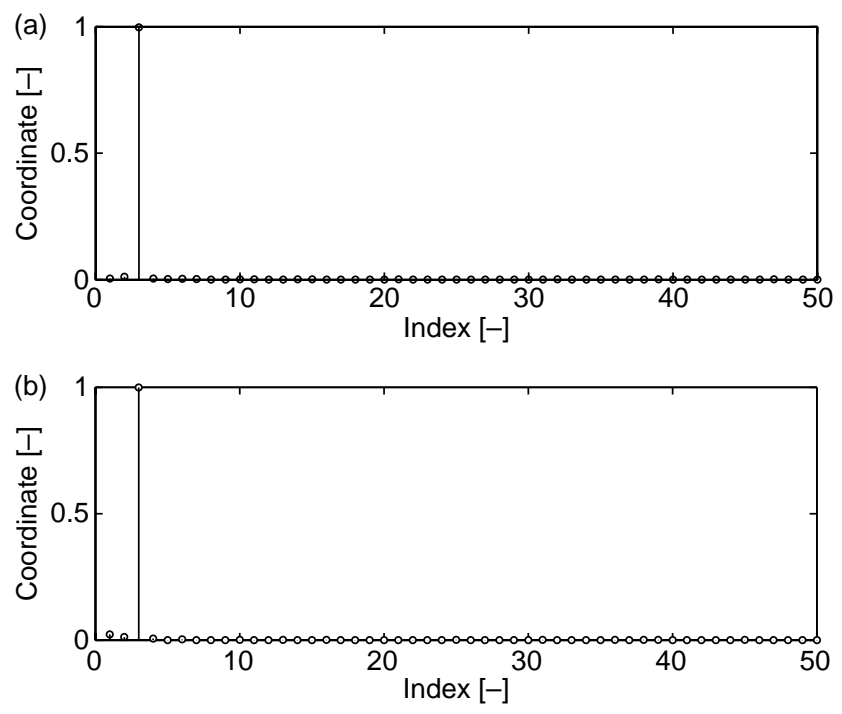

Fig. 14. The first 50 coordinates of two realizations of the third random eigenmode when projected on the basis of deterministic eigenmodes. 

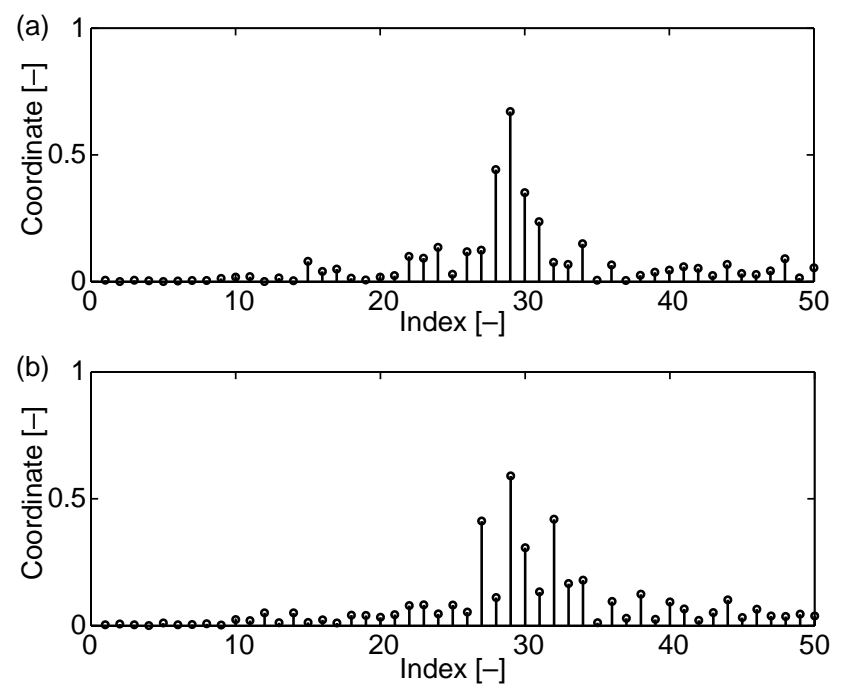

Fig. 15. The first 50 coordinates of two realizations of the 28th random eigenmode when projected on the basis of deterministic eigenmodes.
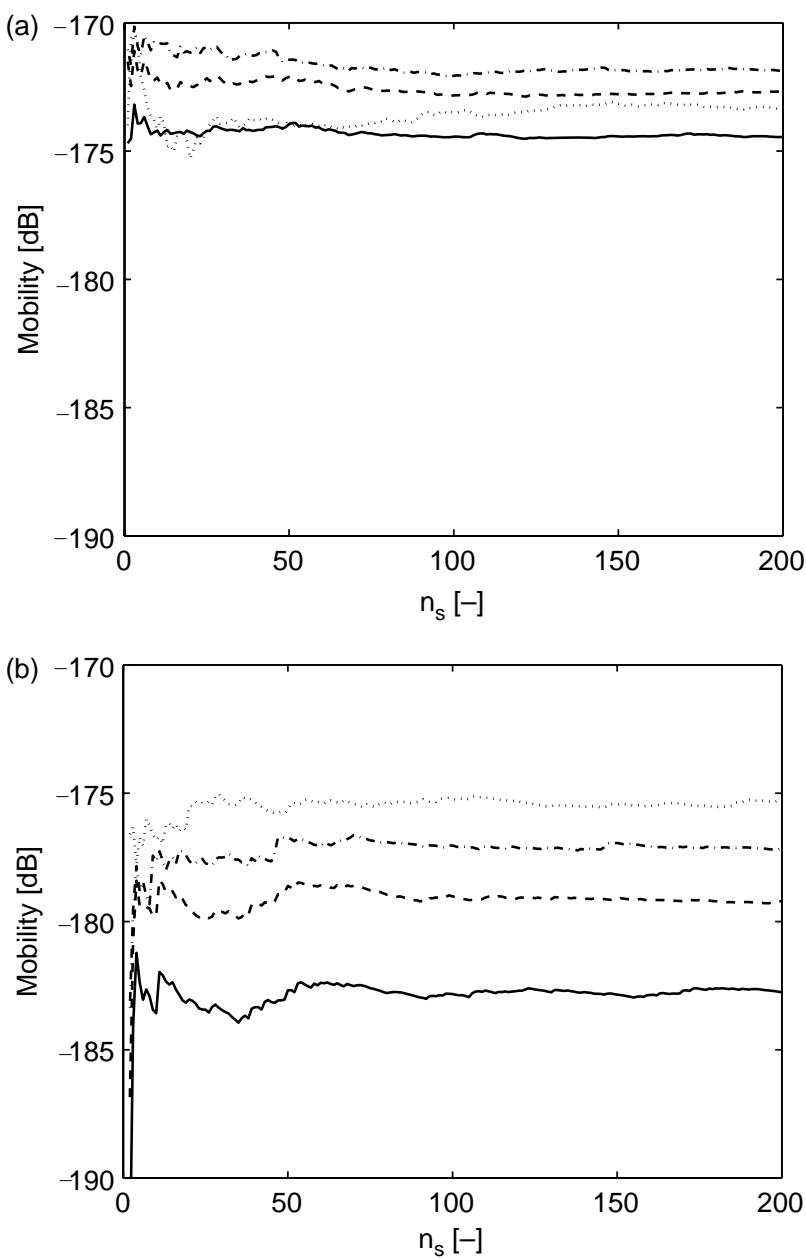

Fig. 16. Estimated (a) mean value and (b) standard deviation of the amplitude of the random FRF (mobility) in the degree of freedom FOPLz as a function of the number of Monte Carlo simulations $n_{s}$ at $50 \mathrm{~Hz}$ for $\delta=0.1$ (solid line), $\delta=0.2$ (dashed line), $\delta=0.3$ (dash-dotted line) and $\delta=0.4$ (dotted line).
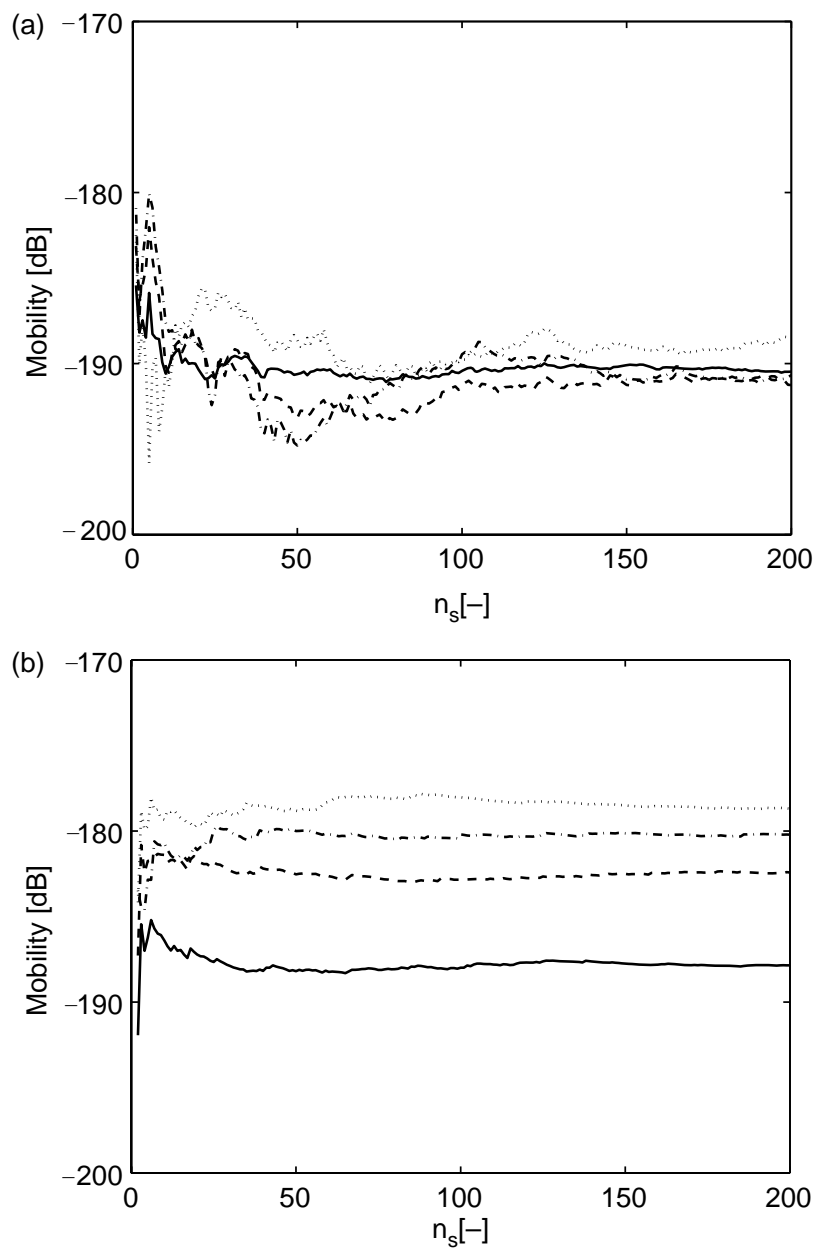

Fig. 17. Estimated (a) mean value and (b) standard deviation of the amplitude of the random FRF (mobility) in the degree of freedom FOPLz as a function of the number of Monte Carlo simulations $n_{s}$ at $100 \mathrm{~Hz}$ for $\delta=$ 0.1 (solid line), $\delta=0.2$ (dashed line), $\delta=0.3$ (dash-dotted line) and $\delta=0.4$ (dotted line).

bending and torsion of the whole building govern the response, the confidence regions for the random FRFs are small. The sensitivity of the low frequency response to uncertainties on the mass and stiffness is small. At higher frequencies, where the response is governed by local plate bending of the floor slabs of the building, the confidence regions are large, even for small values of the dispersion level $\delta$. The sensitivity of the local eigenmodes to small uncertainties on the mass and stiffness involves a large sensitivity of the mid-frequency response to such uncertainties.

Generally, the dispersion of the random response increases as vibrations propagate inside the building. In the degree of freedom $\mathrm{BA01z}$ in the basement of the building, the dispersion of the random response is smaller than in the degrees of freedom FOPLz and F201z, higher in the building and further away from the applied loads. 

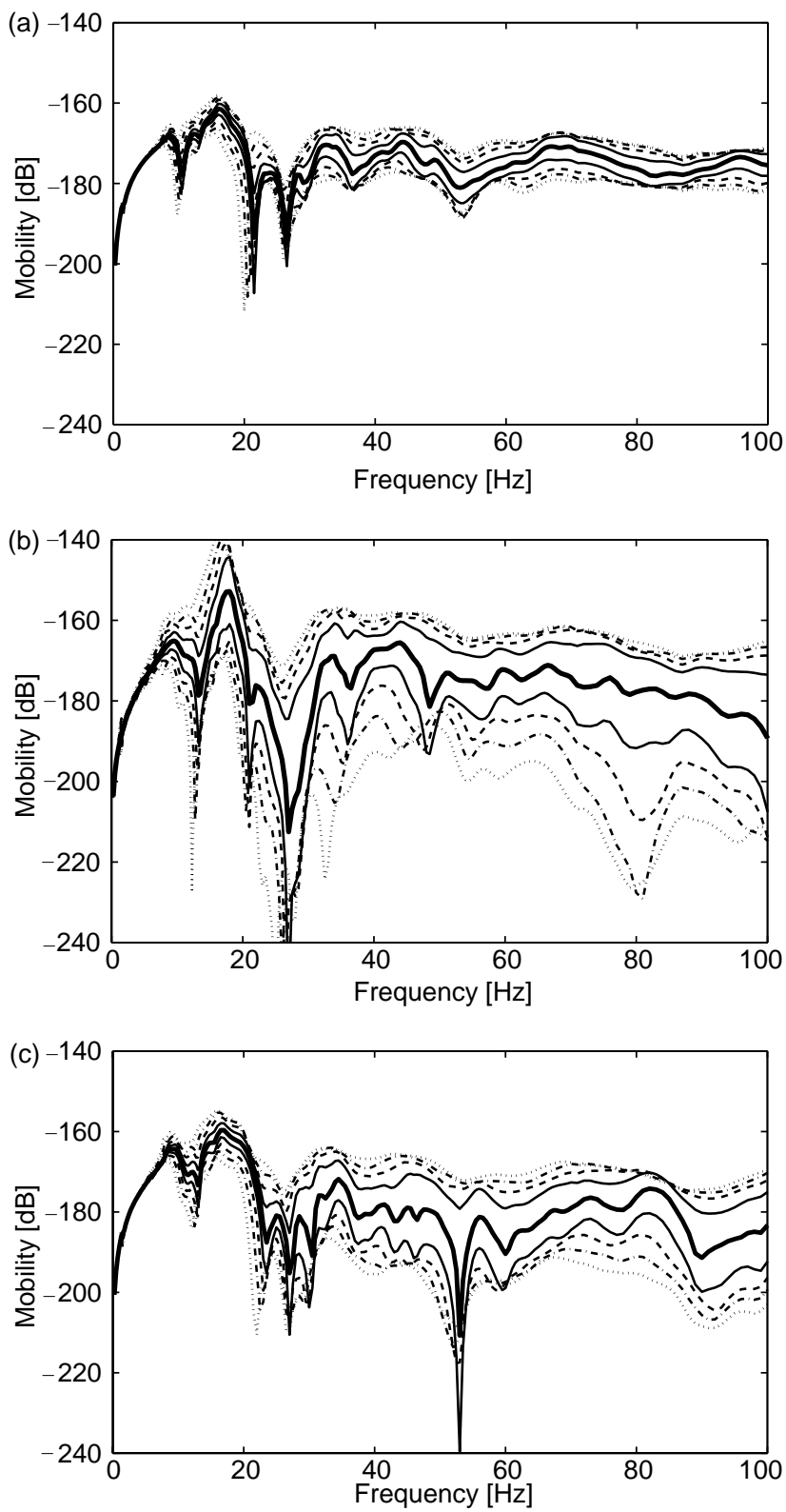

Fig. 18. Estimated bounds of the confidence regions $\left(P_{c}=0.95\right)$ for the random FRFs (mobility) in the degrees of freedom (a) BA01z, (b) F0PLz and (c) F201z for $\delta=0$ (thick solid line), $\delta=0.1$ (solid lines), $\delta=0.2$ (dashed lines), $\delta=0.3$ (dash-dotted lines) and $\delta=0.4$ (dotted lines).

\section{Identification of the dispersion level $\delta$}

In this section, the prediction accuracy of the considered model is quantified. The appropriate dispersion level $\delta$ is identified from the experimental data set. The dispersion level $\delta$ is tuned such that the estimated confidence regions for the random FRFs predicted by the probabilistic model envelope the best estimates for the FRFs, at least where the coherence of the experimental results is good. Due to the low coherence values in the frequency range below $20 \mathrm{~Hz}$ and above $100 \mathrm{~Hz}$, the frequency range for the comparison of the experimental and the modelling results is restricted between 20 and $100 \mathrm{~Hz}$. Based on many simulations, an optimal value $\delta=0.4$ has been identified to best fit the data set.

Figs. 19-21 show the best estimates for the FRFs, the estimated confidence regions for the random FRFs for $\delta=$ 0.4 and the coherence functions between the applied force and the measured response in the degrees of freedom BA01z, F0PLz and F201z. In the basement of the building, the quality of the experimental result BA01z is good. The relatively small confidence region for the random FRF envelopes the best estimate for the FRF. Higher in the building, in the degrees of freedom FOPLz and F201z, the quality of the experimental results is lower. The large confidence regions for the random FRFs envelope the best estimates for the FRFs.

For the response in the degrees of freedom F0PLz and F201z, the obtained confidence regions broadly envelope the best estimates for the FRFs. If only these degrees of freedom had been considered, a smaller dispersion level would have been more appropriate. However, for the degree of freedom BA01z, the identified dispersion level is required for the confidence region to reasonably envelope the best estimate for the FRF. As only a single dispersion parameter is available, the identified value 0.4 must be chosen to fit the whole data set, even if this value is too large for the degrees of freedom higher in the building.

The dispersion of the random FRFs predicted by the probabilistic model for $\delta=0.4$, or the size of the confidence regions for the random FRFs, is used to characterize the prediction accuracy of the model. In the basement, close to the applied loads, the influence of the modelling errors and uncertainties related to the building is relatively small. The size of the confidence region for the random FRF is approximately $10 \mathrm{~dB}$. Higher in the building and further away from the applied loads, this influence is more important. The size of the confidence regions for the random FRFs in the degrees of freedom at the ground and second floor is approximately $20-30 \mathrm{~dB}$.

\section{Conclusions and directions for future work}

In this paper, the prediction accuracy of models for ground-borne vibration in buildings based on a threedimensional coupled FE-BE formulation has been studied. It has been shown that the non-parametric probabilistic approach allows to estimate the prediction accuracy of such models when both parameter uncertainties and modelling errors are present in the building model.

The advantages over so called parametric probabilistic approaches are that the description of the parameter uncertainties in terms of random fields is not required and that both the parameter uncertainties and the modelling errors are incorporated into the analysis. Nevertheless, the non-parametric probabilistic approach is a global approach. It only gives information about the global influence of all 

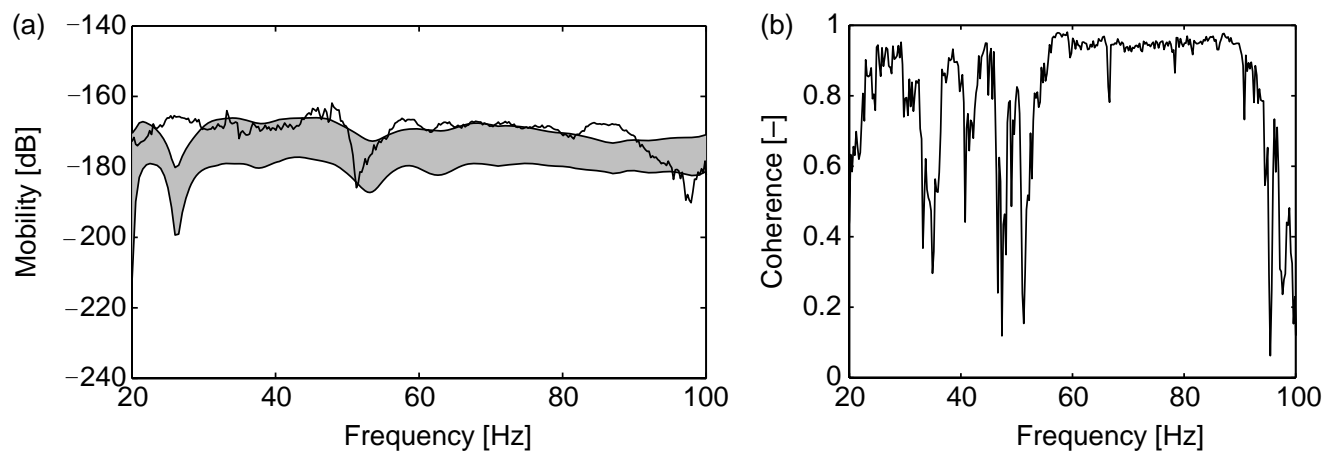

Fig. 19. (a) The best estimate for the FRF (mobility) (thick solid line) and the estimated confidence region for the random FRF for $\delta=0.4$ (grey zone) and (b) coherence function between the applied force and the measured response in the degree of freedom BA01z.
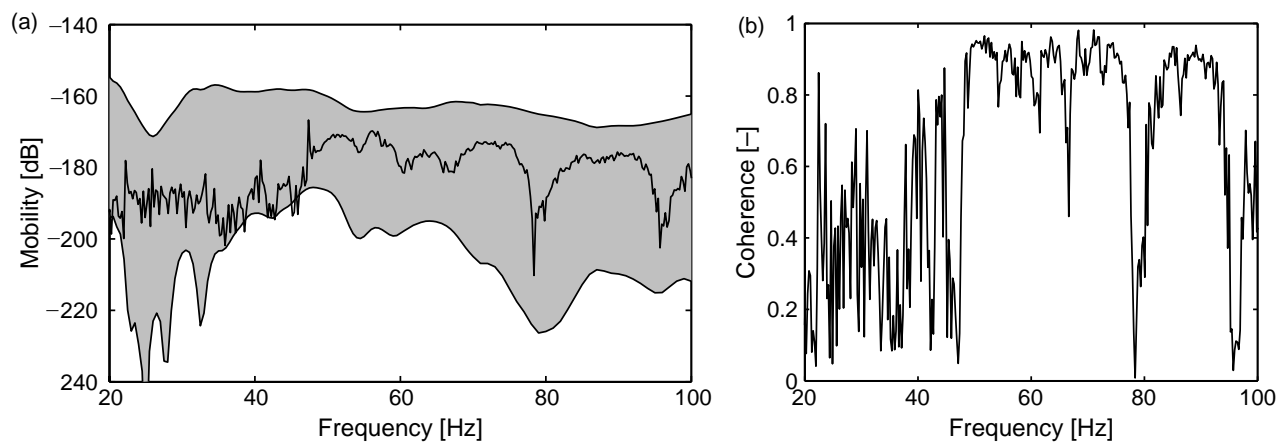

Fig. 20. (a) The best estimate for the FRF (mobility) (thick solid line) and the estimated confidence region for the random FRF for $\delta=0.4$ (grey zone) and (b) coherence function between the applied force and the measured response in the degree of freedom F0PLz.
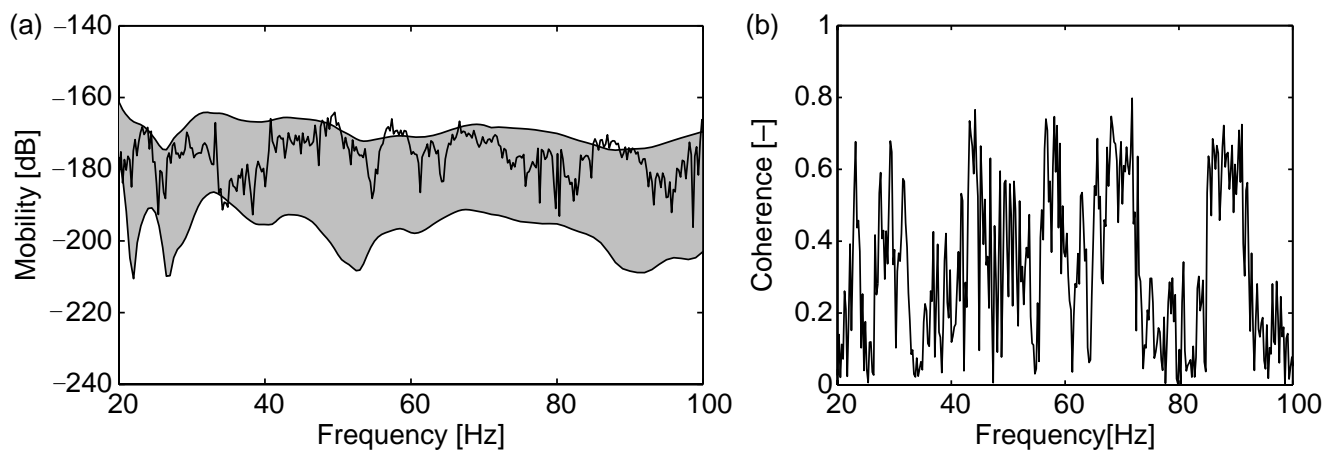

Fig. 21. (a) The best estimate for the FRF (mobility) (thick solid line) and the estimated confidence region for the random FRF for $\delta=0.4$ (grey zone) and (b) coherence function between the applied force and the measured response in the degree of freedom F201z.

the parameter uncertainties and the modelling errors on the model predictions. It does not allow identifying the different sources of the modelling errors and does not provide quantitative information on the parameter uncertainties.

The case history has shown that the approach provides valuable insight as well as quantitative estimations of the prediction accuracy of the considered model. First, the effectiveness of the approach as a tool to investigate the sensitivity of model predictions to arbitrary random uncertainties has been shown. It has been demonstrated that the prediction accuracy decreases with increasing frequency and with increasing distance to the applied loads. Especially on the slabs of the frame structure, a low prediction accuracy has been found. This has been explained by the presence of densely packed clusters of local plate bending eigenmodes in the relevant eigenfrequency range. In this case, small perturbations of the mass or stiffness significantly alter the local eigenmodes, which involves modifications of the response. Because of this physical reason, accurate predictions of the response are very difficult unless parameter uncertainties and modelling errors are sufficiently reduced. Next, the approach has been followed to quantify the prediction accuracy of the considered model. The main difficulty is the identification of the model. In this paper, the non-parametric probabilistic model has been parameterized by a single dispersion 
parameter that has been identified based on graphical comparisons of confidence regions for the random response with experimental results.

The parameterization of the probabilistic model by a single dispersion parameter may lead to confidence regions that are too large in certain degrees of freedom. A first direction for future work may consist of the parameterization of the probabilistic model by a set of parameters rather than by a single dispersion parameter, allowing finer tuning of the model. However, when the probabilistic model is parameterized by more parameters, its identification based on graphical comparisons with experimental results rapidly becomes infeasible.

The development of theoretical estimators for the identification of the probabilistic model from experimental results therefore naturally suggests itself. The main advantage of theoretical estimators is that they allow efficient parameter identification by numerical optimization algorithms, rendering the identification of a probabilistic model parameterized by a set of parameters feasible. The second advantage is that they allow to rigorously account for experimental uncertainties, for example by weighting a distance measure between the experimental and the model results by the coherence function.

A third direction for future work may be to extend the existing non-parametric theory such that a random matrix can substitute the soil impedance matrix as well. This allows studying the influence of parameter uncertainties and modelling errors related to the soil domain. The probability distribution for this random matrix might also be derived from the entropy optimization principle, but in this case, a supplementary constraint must be introduced, namely the causality principle.

\section{Acknowledgements}

The results presented in this paper have been obtained within the frame of the EC-Growth project G3RD-CT-200000381 CONVURT ('The CONtrol of Vibration from Underground Railway Traffic'). Part of the work has been performed in the framework of a BDI scholarship provided by the CNRS. The financial support of the European Community and of the CNRS is duly acknowledged.

\section{References}

[1] Lombaert G, Degrande G. The experimental validation of a numerical model for the prediction of the vibration in the free field produced by road traffic. J Sound Vibr 2003;262: 309-31.

[2] Degrande G, Lombaert G. An efficient formulation of Krylov's prediction model for train induced vibration based on the dynamic reciprocity theorem. J Acoust Soc Am 2001;110(3):1379-90.
[3] Clouteau D, Arnst M, Al-Hussaini TM, Degrande G. Freefield vibrations due to dynamic loading on a tunnel embedded in a stratified medium. J Sound Vibr 2005;238(1-2):173-99.

[4] Craig RR, Bampton CC. Coupling of substructures for dynamic analysis. AIAA J 1968;6(7):1313-9.

[5] Aubry D, Clouteau D. A subdomain approach to dynamic soilstructure interaction. In: Davidovici V, Clough R, editors. Recent advances in earthquake engineering and structural dynamics. Nantes: OUEST Editions/AFPS; 1992. p. 251-72.

[6] Clouteau D, Aubry D. A subdomain approach to dynamic soilstructure interaction. In: Hall WS, Oliveto G, editors. Boundary element methods for soil-structure interaction. Springer; 2003. p. 61-125.

[7] Friswell MJ, Mottershead JE. Finite element model updating in structural dynamics. Dordrecht, The Netherlands: Kluwer; 1995.

[8] Gibert P. Low and medium frequencies in highly heterogeneous structures. Comptes Rendus de l'Académie des Sciences, Série II 1982;295:951-4.

[9] Morand HJ-P, Ohayon R. Fluid structure interaction. New York: Wiley; 1995.

[10] Balmès E. High modal density, curve veering, localization: a different perspective on the structural response. J Sound Vibr 1993;161(2): $358-63$.

[11] Savin E. Midfrequency vibrations of a complex structure: experiments and comparison with numerical simulations. AIAA J 2002;40(9): 1876-84.

[12] Adhikari S, Manohar S. Dynamic analysis of frame structures with statistical uncertainties. Int J Numer Methods Eng 1999;44:1157-78.

[13] Dey S, Shirron JJ, Couchman S. Mid-frequency structural acoustic and vibration analysis in arbitrary, curved three-dimensional domains. Comput Struct 2001;79(6):617-29.

[14] Ben Dhia H. Numerical modeling of multiscale mechanical problems: the Arlequin method. Comptes Rendus de l'Académie des Sciences, Série IIb 1998;326(12):899-904.

[15] Langley RS, Bremner P. A hybrid method for the vibration analysis of complex structural-acoustic systems. J Acoust Soc Am 1999;105(3): $1657-71$.

[16] Soize C. Reduced models in the medium frequency range for general dissipative structural-dynamics systems. Eur J Mech A-Solids 1998; 17(4):657-85.

[17] Sarkar A, Ghanem R. Mid-Frequency structural dynamics with parameter uncertainty. Comput Methods Appl Mech Eng 2002;191: 5499-513.

[18] Ibrahim RA. Structural dynamics with parameter uncertainties. ASME Appl Mech Rev 1987;40(3):309-28.

[19] Manohar CS, Ibrahim RA. Progress in structural dynamics with stochastic parameter variations 1987-1998. ASME Appl Mech Rev 1999;52(5):177-97.

[20] Ghanem R, Spanos P. Stochastic finite elements: a spectral approach.: Springer; 1991.

[21] Mace BR, Shorter J. A local modal/perturbational method for estimating frequency response statistics of built-up structures with uncertain properties. J Sound Vibr 2001;242(5):793-811.

[22] Chen SH, Qiu ZP, Liu S. A method for computing eigenvalue bounds in structural vibration systems with interval parameters. Comput Struct 1994;51(3):309-13.

[23] Soize C. Maximum entropy approach for modeling random uncertainties in transient elastodynamics. J Acoust Soc Am 2001; 109(5):1979-96.

[24] Soize C. A non-parametric model of random uncertainties for reduced matrix models in structural dynamics. Probabil Eng Mech 2000;15(3): 277-94.

[25] Jaynes ET. Information theory and statistical mechanics. Phys Rev 1957;106(4):620-30.

[26] Kapur JN, Kesavan HK. Entropy optimisation principles with applications. San Diego: Academic Press; 1992. 
[27] Chebli H. Modélisation des incertitudes aléatoires non homogènes en dynamique des structures pour le domaine des basses fréquences. $\mathrm{PhD}$ thesis, Conservatoire Nationale des Arts et Métiers, France, 2002.

[28] Pyl L, Degrande G. Determination of the dynamic soil characteristics with the SASW method at the site of Cité Universitaire in Paris. Report BWM-2002-08, Department of Civil Engineering K.U.Leuven, October 2002. CONVURT EC-Growth Project G3RDCT-2000-00381.

[29] Chatterjee P, Degrande G, Jacobs S, Charlier J, Bouvet P, Brassenx D. Experimental results of free field and structural vibrations due to underground railway traffic. In: 10th international congress on sound and vibration, Stockholm, Sweden, July 2003.
[30] Coster C. Vibrations due to underground railway traffic. Master's thesis, Ecole Supérieure de Mécanique de Marseille and Department of Civil Engineering, K.U. Leuven, France; 2001.

[31] Arnst M. Three-dimensional modelling of free field and structural vibration due to harmonic and transient loading in a tunnel. Master's thesis, Department of Civil Engineering, K.U. Leuven and Ecole Centrale Paris, Belgium; 2003.

[32] Heylen W, Lammens S, Sas P. Modal analysis theory and testing. Leuven, Belgium: K.U. Leuven Press; 1995.

[33] Balmès E. Structural dynamics toolbox, user's guide, version 5.1. Scientific Software Group, 2004. 